\title{
Legendrian knots, transverse knots and combinatorial Floer homology
}

\author{
PETER OZSVÁth \\ ZOLTÁn SZABó \\ DYLAN THURSTON
}

\begin{abstract}
Using the combinatorial approach to knot Floer homology, we define an invariant for Legendrian knots (or links) in the three-sphere, with values in knot Floer homology. This invariant can also be used to construct an invariant of transverse knots.
\end{abstract}

53D12, 57R17, 57R58; 57M25

\section{Introduction}

We use link Floer homology to study contact phenomena for links in the three-sphere, endowed with its standard contact structure.

Knot (or link) Floer homology is an invariant defined using Heegaard diagrams and holomorphic disks, as in Oszváth and Szabó [15] and Rasmussen [21]. It comes in various forms, but the version which will be of primary interest to us here is the variant which associates to a knot $K$ a module over $\mathbb{Z}[U]$, denoted

$$
H F K^{-}(K)=\bigoplus_{m, a \in \mathbb{Z}} H F K_{m}^{-}(K, a),
$$

where $U$ acts as an endomorphism which is homogeneous of degree -2 for the Maslov grading $m$ and degree -1 for the Alexander grading $a$.

Manolescu, Ozsváth and Sarkar gave [9] an explicit description of knot Floer homology for a knot in the three-sphere as the homology groups of a chain complex $\mathrm{CK}^{-}$which is described in terms of the combinatorics of a grid diagram for a knot. In fact, the constructions of [9] are done with coefficients in $\mathbb{Z} / 2 \mathbb{Z}$; a lift of these constructions to coefficients in $\mathbb{Z}$ is given by Manolescu and the authors [10], along with a purely combinatorial proof of the fact that their homology groups are knot invariants, which entirely circumvents the holomorphic description. Given a grid diagram $G$ for the mirror $m(K)$ of a knot $K$, we refer to the resulting complex as the combinatorial chain 
complex for $G$, denoted $C K^{-}(G)$. Specifically, $C K^{-}(G)$ is generated by a collection of generators $\mathbf{S}$, equipped with grading functions $A$ and $M$, so that

$$
C K^{-}(G)=\bigoplus_{m, a \in \mathbb{Z}} C K_{m}^{-}(G, a)
$$

where $C K_{m}^{-}(G, a)$ denotes the set of $\mathbb{Z}$-linear combinations of generators $\mathbf{x}$ with $A(\mathbf{x})=a$ and $M(\mathbf{x})=m . C K^{-}(G)$ has an explicitly defined differential $\partial^{-}$, with $\partial^{-}: C K_{m}^{-}(G, a) \rightarrow C K_{m-1}^{-}(G, a)$ (so the bigrading descends to homology). On the other hand, a grid diagram may also be used to give a combinatorial presentation of a Legendrian knot $\mathcal{K}$ or a transverse knot $\mathcal{T}$; we will exploit this fact here. (By our conventions, explained in Section 4, the topological type of $\mathcal{K}$ is the mirror of $K$.)

Given a grid diagram for a knot, we will exhibit a concrete pair of generators $z^{+}$and $z^{-}$ for the combinatorial chain complex, both of which are cycles. These elements are defined in Definitions 6.1 and 6.10, and the fact they are cycles is established in Lemma 6.2. Our aim here is to study this pair of cycles. We show that the pair of induced homology classes is an invariant for Legendrian knots. To describe the bigradings of these elements, we use the two classical invariants of a Legendrian knot $\mathcal{K}$, the Thurston-Bennequin invariant $\mathrm{tb}(\mathcal{K})$ and the rotation number $\mathrm{r}(\overrightarrow{\mathcal{K}})$, which we recall in Section 2. The overall sign of the rotation number depends on the orientation of $\overrightarrow{\mathcal{K}}$. (Note that we restrict our attention in this introduction, and indeed through most of the present paper, to the case of knots, as opposed to links, though most of the results here carry over with minor modifications to the case of links. These generalizations are discussed in Section 7.)

Theorem 1.1 For a grid diagram $G$ which represents a knot, let $\overrightarrow{\mathcal{K}}=\overrightarrow{\mathcal{K}}(G)$ be the corresponding oriented Legendrian knot. Then there are two associated cycles $z^{+}=z^{+}(G)$ and $z^{-}=z^{-}(G)$, supported in bigradings

$$
\begin{aligned}
M\left(z^{+}\right) & =\operatorname{tb}(\overrightarrow{\mathcal{K}})-\mathrm{r}(\overrightarrow{\mathcal{K}})+1, & & M\left(z^{-}\right)=\operatorname{tb}(\overrightarrow{\mathcal{K}})+\mathrm{r}(\overrightarrow{\mathcal{K}})+1, \\
A\left(z^{+}\right) & =\frac{\operatorname{tb}(\overrightarrow{\mathcal{K}})-\mathrm{r}(\overrightarrow{\mathcal{K}})+1}{2}, & & A\left(z^{-}\right)=\frac{\operatorname{tb}(\overrightarrow{\mathcal{K}})+\mathrm{r}(\overrightarrow{\mathcal{K}})+1}{2} .
\end{aligned}
$$

Moreover, if $G$ and $G^{\prime}$ are two different grid diagrams which represent Legendrian isotopic oriented knots, then there is a quasi-isomorphism of chain complexes

$$
\Phi: C K^{-}(G) \longrightarrow C K^{-}\left(G^{\prime}\right)
$$

with

$$
\Phi\left(z^{+}(G)\right)=z^{+}\left(G^{\prime}\right), \quad \Phi\left(z^{-}(G)\right)=z^{-}\left(G^{\prime}\right) .
$$

If $G$ is a grid diagram representing an oriented Legendrian knot $\overrightarrow{\mathcal{K}}$, then we denote the homology classes of $z^{+}(G)$ and $z^{-}(G)$ in $H F K^{-}(m(K))$ by $\lambda^{+}(\overrightarrow{\mathcal{K}})$ and $\lambda^{-}(\overrightarrow{\mathcal{K}})$ 
respectively, and refer to them as the Legendrian invariants of $\overrightarrow{\mathcal{K}}$. The pair of invariants $\lambda^{+}(\overrightarrow{\mathcal{K}})$ and $\lambda^{-}(\overrightarrow{\mathcal{K}})$ can be used to distinguish Legendrian knots with the same classical invariants. For example, for the two different Legendrianizations of the knot $5_{2}, \overrightarrow{\mathcal{K}}_{1}$ and $\overrightarrow{\mathcal{K}}_{2}$ with $\mathrm{r}=0$ and tb $=1$, we have that $\lambda^{+}\left(\overrightarrow{\mathcal{K}}_{1}\right) \neq \lambda^{-}\left(\overrightarrow{\mathcal{K}}_{1}\right)$, while $\lambda^{+}\left(\overrightarrow{\mathcal{K}}_{2}\right)=\lambda^{-}\left(\overrightarrow{\mathcal{K}}_{2}\right)$. See Example 8.1 below.

There is some symmetry in the construction of the cycles $z^{+}$and $z^{-}$. To this end, recall the Legendrian mirror construction; see $\mathrm{Ng}$ [11] and Fuchs and Tabachnikov [7]. Given an oriented Legendrian knot $\overrightarrow{\mathcal{K}}$, one can rotate the front projection by $180^{\circ}$ around the $x$-axis to obtain the Legendrian knot projection of an oriented Legendrian knot denoted $\mu(\overrightarrow{\mathcal{K}})$. Classical invariants are related by

$$
\operatorname{tb}(\mu(\overrightarrow{\mathcal{K}}))=\operatorname{tb}(\overrightarrow{\mathcal{K}}), \quad \operatorname{rr}(\mu(\overrightarrow{\mathcal{K}}))=-\mathrm{r}(\overrightarrow{\mathcal{K}}) .
$$

One can instead reverse the orientation of $\overrightarrow{\mathcal{K}}$ to obtain a different oriented Legendrian knot $-\overrightarrow{\mathcal{K}}$ with

$$
\operatorname{tb}(-\overrightarrow{\mathcal{K}})=\operatorname{tb}(\overrightarrow{\mathcal{K}}), \quad \quad \mathrm{r}(-\overrightarrow{\mathcal{K}})=-\mathrm{r}(\overrightarrow{\mathcal{K}}) .
$$

Proposition 1.2 Suppose that $G$ is a grid representation of $\overrightarrow{\mathcal{K}}, G_{1}$ is a grid representation of the orientation reversal $-\overrightarrow{\mathcal{K}}$, and $G_{2}$ is a grid representation of the Legendrian mirror $\mu(\overrightarrow{\mathcal{K}})$. Then there are quasi-isomorphisms

$$
\Phi_{1}: C K^{-}(G) \longrightarrow C K^{-}\left(G_{1}\right), \quad \Phi_{2}: C K^{-}(G) \longrightarrow C K^{-}\left(G_{2}\right)
$$

which have the property that

$$
\begin{array}{llrl}
\Phi_{1}\left(z^{+}(G)\right)=z^{-}\left(G_{1}\right), & \Phi_{2}\left(z^{+}(G)\right)=z^{-}\left(G_{2}\right), \\
\Phi_{1}\left(z^{-}(G)\right)=z^{+}\left(G_{1}\right), & \Phi_{2}\left(z^{-}(G)\right)=z^{+}\left(G_{2}\right) .
\end{array}
$$

More interestingly, this invariant behaves in a controlled manner under stabilizations of the Legendrian knot. Specifically, recall that one can locally introduce a pair of cusps in the front projection of a Legendrian knot $\mathcal{K}$ to obtain a new Legendrian knot $\mathcal{K}^{\prime}$ which is in the same topological type as $\mathcal{K}$, but which is not Legendrian isotopic to the original knot. The knot $\mathcal{K}^{\prime}$ is called a stabilization of $\mathcal{K}$. If we fix an orientation for $\mathcal{K}$, we can distinguish the two ways of stabilizing as positive and negative. (We adhere to the conventions spelled out, for example, in Etnyre's survey [5], which, incidentally, is also an excellent reference for the basic theory of Legendrian and transverse knots. We review these conventions in Section 2.) The Legendrian invariants transforms in the following manner under stabilizations: 
Theorem 1.3 Let $\overrightarrow{\mathcal{K}}$ be an oriented Legendrian knot, and $\overrightarrow{\mathcal{K}}^{-}$(respectively $\overrightarrow{\mathcal{K}}^{+}$) be the oriented Legendrian knots obtained as a single negative (respectively positive) stabilization of $\overrightarrow{\mathcal{K}}$. Then there are quasi-isomorphisms

$$
\Phi^{-}: C K^{-}(\overrightarrow{\mathcal{K}}) \longrightarrow C K^{-}\left(\overrightarrow{\mathcal{K}}^{-}\right), \quad \Phi^{+}: C K^{-}(\overrightarrow{\mathcal{K}}) \longrightarrow C K^{-}\left(\overrightarrow{\mathcal{K}}^{+}\right)
$$

under which

$$
\begin{aligned}
\phi^{-}\left(\lambda^{+}(\overrightarrow{\mathcal{K}})\right) & =\lambda^{+}\left(\overrightarrow{\mathcal{K}}^{-}\right), & U \cdot \phi^{+}\left(\lambda^{+}(\overrightarrow{\mathcal{K}})\right) & =\lambda^{+}\left(\overrightarrow{\mathcal{K}}^{+}\right), \\
U \cdot \phi^{-}\left(\lambda^{-}(\overrightarrow{\mathcal{K}})\right) & =\lambda^{-}\left(\overrightarrow{\mathcal{K}}^{-}\right), & \phi^{+}\left(\lambda^{-}(\overrightarrow{\mathcal{K}})\right) & =\lambda^{-}\left(\overrightarrow{\mathcal{K}}^{+}\right),
\end{aligned}
$$

where $\phi^{ \pm}$denotes the map induced on homology by $\Phi^{ \pm}$.

The above theorem suggests an application to transverse knots. A transverse knot $\mathcal{T}$ inherits its orientation from the contact structure on $S^{3}$. If we let $\overrightarrow{\mathcal{K}}$ be a Legendrian approximation to $\mathcal{T}$ (see Section 2) with the natural induced orientation, then we can define the transverse invariant $\theta(\mathcal{T})$ to be $\lambda^{+}(\overrightarrow{\mathcal{K}})$. By a result of Epstein, Fuchs and Meyer [4] (generalized by Etnyre and Honda [6]), any two Legendrian approximations to some given transverse knot are equal after some number of negative stabilizations. Thus, in view of Theorem 1.3, we can conclude that the transverse invariant is independent of the choice of Legendrian approximation $\overrightarrow{\mathcal{K}}$ used in its definition.

Corollary 1.4 The transverse invariant $\theta(\mathcal{T})$ depends only on the transverse isotopy class of the transverse knot $\mathcal{T}$; ie, if $G$ and $G^{\prime}$ are two grid diagrams representing two Legendrian approximations to $\mathcal{T}$, then there is a quasi-isomorphism

$$
\Phi: C K^{-}(G) \longrightarrow C K^{-}\left(G^{\prime}\right)
$$

whose induced map $\phi$ on homology has the property that $\phi(\theta(\mathcal{T}))=\theta\left(\mathcal{T}^{\prime}\right)$.

In later work by $\mathrm{Ng}$, Ozsváth and Thurston, the invariant $\theta$ is used to distinguish particular transversally nonisotopic knots with the same classical invariants [13].

These invariants also satisfy the following nonvanishing property.

Theorem 1.5 For any Legendrian knot $\overrightarrow{\mathcal{K}}$, the homology classes $\lambda^{+}(\overrightarrow{\mathcal{K}})$ and $\lambda^{-}(\overrightarrow{\mathcal{K}})$ are nontrivial; and they are not $U$-torsion classes (ie, for all positive integers $d, U^{d}$ times these classes is nontrivial). Similarly, for any transverse knot $\mathcal{T}$, the transverse invariant $\theta(\mathcal{T})$ does not vanish, and indeed is not $U$-torsion. 
We can use this to reprove bounds on the Thurston-Bennequin invariant. Recall that $\tau(K) \in \mathbb{Z}$ is a knot concordance invariant defined using $\operatorname{HFK}^{-}(K)$ [14]. One definition is that $\tau(m(K))$ is the minimal Alexander grading of any element $\xi$ of $H F K^{-}(K)$ for which $U^{d} \xi \neq 0$ for all integers $d \geq 0$. (This is not the usual definition of $\tau$, but we verify in Appendix A that it is equivalent.) Combining Theorem 1.5 with Theorem 1.1, we see at once that for any Legendrian knot $\mathcal{K}$,

$$
|\mathrm{r}(\mathcal{K})|+\operatorname{tb}(\mathcal{K}) \leq 2 \tau(\mathcal{K})-1
$$

a bound which was first proved by Plamenevskaya [18] using the contact invariant in Heegaard Floer homology [16]. The inequality $\tau(K) \leq g(K)$ is easy to establish [15], giving another proof of Bennequin's inequality. Indeed, since $\tau(K) \leq g^{*}(K)$ [14], we have yet another proof of the "slice-Bennequin inequality"

$$
|\mathrm{r}(\mathcal{K})|+\operatorname{tb}(\mathcal{K}) \leq 2 g^{*}(K)-1,
$$

first proved using methods of gauge theory; see Rudolph [22] and Kronheimer and Mrowka [8].

It is interesting to compare our results here with those of Plamenevskaya [19]. In that paper, Plamenevskaya uses braid representatives to give an invariant for transverse knots which takes values in the Khovanov homology of the knot. She uses this to prove the following analogue of Equation (1):

$$
|\mathrm{r}(\mathcal{K})|+\operatorname{tb}(\mathcal{K}) \leq s(K)-1,
$$

where now $s(K)$ is the Rasmussen invariant coming from Khovanov homology [20]. This bound, when combined with Rasmussen's bound $s(K) \leq 2 g^{*}(K)$, gives a different proof of the slice-Bennequin inequality. ( $\mathrm{Ng}$ gives a different Legendrian ThurstonBennequin bound using Khovanov homology [12].)

In Section 2, we review some conventions on Legendrian and transverse knots. In Section 3, we recall the combinatorial chain complex from a grid diagram [9]. In Section 4, we describe the relationship between grid diagrams, Legendrian knots, and their stabilizations. In Section 5, we recall the isomorphisms on homology induced by basic moves on grid diagrams [10]. The Legendrian invariant is defined in Section 6, and the properties stated above are established there. In this introduction, and indeed throughout most of this paper, we have focused on the case of knots, rather than links, mainly for notational simplicity. In Section 7, we extend the results to the case of links in $S^{3}$. Finally, in Section 8 we give examples of the computation of the Legendrian elements. We reprove that the knot $5_{2}$ is not Legendrian simple, and show that the link $6_{3}^{2}$ is not, either.

Geometry 83 Topology, Volume 12 (2008) 
Acknowledgements The authors wish to thank John Baldwin, John Etnyre, Ciprian Manolescu, Lenhard Ng, Jacob Rasmussen, and András Stipsicz for helpful discussions during the course of this work. We are especially indebted to $\mathrm{Ng}$ for sharing with us his expertise and clarifying some questions about symmetries of Legendrian knots. Moreover, it appears that Baldwin and $\mathrm{Ng}$ have both independently discovered at least some of the structures described here.

PO was supported by NSF grant number DMS-0505811 and FRG-0244663. ZSz was supported by NSF grant number DMS-0406155 and FRG-0244663. DT was supported by a Sloan Research Fellowship.

\section{Legendrian and transverse knots}

We first recall some standard definitions from contact topology. Etnyre's survey [5] is a good reference for this material.

Endow $S^{3}$ with its standard contact structure $\xi$. Restricted to $\mathbb{R}^{3} \subset S^{3}$, this contact structure is the two-plane distribution which is the kernel of the one-form $d z-y d x$.

Recall that a Legendrian knot is a smooth knot $\mathcal{K} \subset S^{3}$ whose tangent vectors are contained in the contact planes of $\xi$. Two knots are Legendrian isotopic if they can be connected by a smooth one-parameter family of Legendrian knots.

There are two classical invariants of Legendrian knots, the rotation number and the Thurston-Bennequin invariant, as follows.

Fix an embedded Seifert surface $F$ for the oriented Legendrian knot $\overrightarrow{\mathcal{K}}$. The restriction of $\xi$ to $F$ determines an oriented two-plane bundle over $F$, which has a trivialization along the boundary induced by tangent vectors to the knot. The rotation number $\mathrm{r}(\overrightarrow{\mathcal{K}})$ is the relative first Chern number of this two-plane field over $F$, relative to the trivialization over $\partial F$.

On the other hand, the restriction of $\xi$ to $\mathcal{K}$ determines a framing of $\mathcal{K}$. The ThurstonBennequin number $\operatorname{tb}(\mathcal{K})$ is the self-linking number of $\overrightarrow{\mathcal{K}}$ with respect to this framing. That is, if we let $\overrightarrow{\mathcal{K}}^{\prime}$ denote a pushoff of $\overrightarrow{\mathcal{K}}$ with respect to the framing, the ThurstonBennequin invariant is the oriented intersection number of $\overrightarrow{\mathcal{K}}^{\prime}$ with $F$.

Note that the overall sign of the rotation number depends on the choice of orientation for $\overrightarrow{\mathcal{K}}$, but the Thurston-Bennequin invariant is independent of this choice.

It is customary to study Legendrian knots via their front projections, defined by the projection map $(x, y, z) \mapsto(x, z)$. The front projection of a Legendrian embedding has 
no vertical tangencies, and in the generic case, its only singularities are double-points and cusps.

The classical invariants of (oriented) Legendrian knots $\overrightarrow{\mathcal{K}}$ can be read off from their (oriented) front projections $\Pi$; indeed, we have

$$
\begin{aligned}
\operatorname{tb}(\mathcal{K}) & =\operatorname{wr}(\Pi)-\frac{1}{2} \#\{\text { cusps in } \Pi\}, \\
\operatorname{r}(\overrightarrow{\mathcal{K}}) & =\frac{1}{2}(\#\{\text { downward-oriented cusps }\}-\#\{\text { upward-oriented cusps }\}),
\end{aligned}
$$

where here $\operatorname{wr}(\Pi)$ denotes the writhe of the projection, ie, the number of positive minus the number of negative crossings of the projection.

A transverse knot is a knot $\mathcal{T} \subset S^{3}$ is a knot whose tangent vectors are transverse to the contact planes of $\xi$. Two transverse knots are transverse isotopic if they can be connected by a smooth one-parameter family of transverse knots.

There is a classical invariant for transverse knots $\mathcal{T}$, the self-linking number, $\operatorname{sl}(\mathcal{T})$. To define this, observe first that since $\xi$ is cooriented, transverse knots inherit a canonical orientation: the orientation for which the intersection number of $\gamma^{\prime}(t)$ with the plane $\xi_{\gamma(t)}$ is positive for all $t \in[0,1]$ (or, equivalently, the evaluation of $d z-y d x$ is positive on a vector oriented in the direction of the knot). Next, consider a Seifert surface $F$ for $\mathcal{T}$ compatible with its orientation. Now, along $\mathcal{T}$, the tangent space to $F$ and the contact planes $\xi$ intersect in a line field, which in turn inherits a natural orientation as "outward pointing" along $F$. Thus, we have a trivialization of $\left.\xi\right|_{\partial F}$. The self-linking number, then, is the relative first Chern number of $\xi$ on $F$ relative to this trivialization of $\xi$ along its boundary.

Given an oriented Legendrian knot $\overrightarrow{\mathcal{K}}$, there are arbitrarily close smooth curves (in the $C^{\infty}$ topology) which are transverse (and which inherit the same orientation as $\overrightarrow{\mathcal{K}}$ ). We call such a knot the (positive) transverse pushoff of $\overrightarrow{\mathcal{K}}$. Conversely, a transverse knot has a regular neighborhood which is contactomorphic to the solid torus endowed with a standard contact structure, for which the core is transverse. There are Legendrian curves in this solid torus which are transverse to the meridional disks, meeting each disk in a single, transverse point of intersection. We call these Legendrian curves the Legendrian approximations to the transverse knot $\mathcal{T}$. The transverse pushoff of a Legendrian approximation to $\mathcal{T}$ is transversally isotopic to $\mathcal{T}$, and a Legendrian knot $\overrightarrow{\mathcal{K}}$ becomes Legendrian isotopic, after it is negatively stabilized sufficiently many times, to a Legendrian approximation to its transverse pushoff. If $\mathcal{T}$ is the transverse pushoff of $\overrightarrow{\mathcal{K}}$, then

$$
\operatorname{sl}(\mathcal{T})=\operatorname{tb}(\overrightarrow{\mathcal{K}})-\mathrm{r}(\overrightarrow{\mathcal{K}})
$$



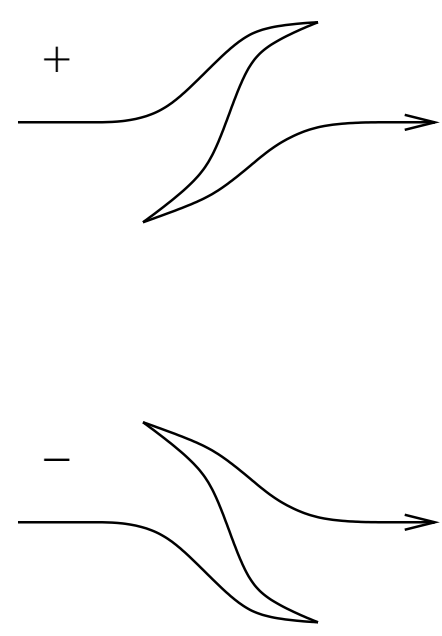

Figure 1: Introducing cusps. Given an oriented arc in an oriented Legendrian knot, we can introduce two cusps locally, in two ways, as pictured. The top is positive stabilization, the bottom is negative stabilization.

Any two Legendrian approximations to a transverse knot can be negatively stabilized so that they become Legendrian isotopic [4]. Thus, Legendrian knots modulo negative stabilization are the same as transverse knots modulo isotopy.

\section{Combinatorial knot Floer homology}

We now recall the combinatorial chain complex for Heegaard Floer homology from earlier papers $[9 ; 10]$, to which the reader is directed for a more in-depth treatment.

Recall that a (planar) grid diagram $G$ lies in a square grid on the plane with $n \times n$ squares. Each square is decorated either with an $X$, an $O$, or nothing, arranged so that:

- every row contains exactly one $X$ and one $O$; and

- every column contains exactly one $X$ and one $O$.

The number $n$ is called the grid number of $G$. We number the $O$ 's and $X$ 's by $\left\{O_{i}\right\}_{i=1}^{n}$ and $\left\{X_{i}\right\}_{i=1}^{n}$, and we denote the two sets by $\mathbb{O}$ and $\mathbb{X}$, respectively. (We use here the notation from [10]; the $O_{i}$ correspond to the "white dots" of [9] and the $w_{i}$ of [17], while the $X_{i}$ to the "black dots" of [9] and the $z_{i}$ of [17].) 
Given a planar grid diagram $G$, we place it in a standard position on the plane by placing the bottom left corner at the origin, and making each cell a square of edge length one. We then construct an oriented, planar link projection by drawing horizontal segments from the $O$ 's to the $X$ 's in each row, and vertical segments from the $X$ 's to the $O$ 's in each column. At every intersection point, we let the horizontal segment be the underpass and the vertical one the overpass. This produces a planar diagram for an oriented link $\vec{L}$ in $S^{3}$. We say that $\vec{L}$ has a grid presentation given by $G$. It is easy to tell if a given grid presentation determines a knot. For the moment, we restrict attention to this case, and return to the more general case in Section 7.

If we cyclically permute the rows or column of a grid diagram, we do not change the link that it represents, so from now on we will think of grid diagrams as being drawn on a torus $T^{2}$. Let the horizontal (respectively vertical) (grid) circles be the circles in between two adjacent rows (respectively columns) of marked squares.

Given a toroidal grid diagram $G$, we associate to it a chain complex $\left(C^{-}\left(G ; \mathbb{F}_{2}\right), \partial^{-}\right)$ as follows. Let $\mathbf{S}=\mathbf{S}(G)$ be the set of one-to-one correspondences between the horizontal and vertical grid circles. More geometrically, we can think of elements of $\mathbf{S}$ as $n$-tuples of intersection points between the horizontal and vertical grid circles, with the property that there is exactly one intersection point on each horizontal and vertical grid circle. Let $C^{-}\left(G ; \mathbb{F}_{2}\right)$ be the free module over $\mathbb{F}_{2}\left[U_{1}, \ldots, U_{n}\right]$ generated by elements of $\mathbf{S}$, where here the $\left\{U_{i}\right\}_{i=1}^{n}$ are indeterminates.

The complex has a bigrading, induced by two functions $A: \mathbf{S} \rightarrow \mathbb{Z}$ and $M: \mathbf{S} \rightarrow \mathbb{Z}$ defined as follows.

Given two collections $A, B$ of finitely many points in the plane, let $\mathcal{I}(A, B)$ be the number of pairs $\left(a_{1}, a_{2}\right) \in A$ and $\left(b_{1}, b_{2}\right) \in B$ with $a_{1}<b_{1}$ and $a_{2}<b_{2}$, and let $\mathcal{J}(A, B)=(\mathcal{I}(A, B)+\mathcal{I}(B, A)) / 2$. Take a fundamental domain for the torus which is cut along a horizontal and vertical circle, with the left and bottom edges included. Given a generator $\mathbf{x} \in \mathbf{S}$, we view $\mathbf{x}$ as a collection of points in this fundamental domain. Similarly, we view $\mathbb{O}=\left\{O_{i}\right\}_{i=1}^{n}$ as a collection of points in the plane. Define

$$
M(\mathbf{x}):=\mathcal{J}(\mathbf{x}, \mathbf{x})-2 \mathcal{J}(\mathbf{x}, \mathbb{O})+\mathcal{J}(\mathbb{O}, \mathbb{O})+1 .
$$

We find it convenient to write this formula more succinctly as

$$
M(\mathbf{x})=\mathcal{J}(\mathbf{x}-\mathbb{O}, \mathbf{x}-\mathbb{O})+1,
$$

where we extend $\mathcal{J}$ bilinearly over formal sums (or differences) of subsets. $M(\mathbf{x})$ depends only on the sets $\mathbf{x}$ and $\mathbb{O}$, but not on how we drew the torus on the plane, as we showed earlier [10, Lemma 2.4]. Furthermore, by the argument there we can 
alternately compute $M$ using a fundamental domain that includes the right and top edges instead of the left and bottom edges.

Define $M_{S}(\mathbf{x})$ to be the same as $M(\mathbf{x})$ with the set $S$ playing the role of $\mathbb{O}$. We define

$$
\begin{aligned}
A(\mathbf{x}) & :=\frac{1}{2}\left(M_{\mathbb{O}}(\mathbf{x})-M_{\mathbb{X}}(\mathbf{x})\right)-\left(\frac{n-1}{2}\right) \\
& =\mathcal{J}\left(\mathbf{x}-\frac{1}{2}(\mathbb{X}+\mathbb{O}), \mathbb{X}-\mathbb{O}\right)-\left(\frac{n-1}{2}\right) .
\end{aligned}
$$

The module $C^{-}\left(G ; \mathbb{F}_{2}\right)$ inherits a bigrading from the functions $M$ and $A$ above, with the additional convention that multiplication by $U_{i}$ drops the Maslov grading by two and the Alexander grading by one.

Given a pair of generators $\mathbf{x}$ and $\mathbf{y}$, and an embedded rectangle $r$ in $T^{2}$ whose edges are arcs in the horizontal and vertical circles, we say that $r$ connects $\mathbf{x}$ to $\mathbf{y}$ if $\mathbf{x}$ and $\mathbf{y}$ agree along all but two horizontal circles, if all four corners of $r$ are intersection points in $\mathbf{x} \cup \mathbf{y}$, and if when we traverse each horizontal boundary component of $r$ in the direction dictated by the orientation that $r$ inherits from $T^{2}$, the arc is oriented from a point in $\mathbf{x}$ to the point in $\mathbf{y}$. Let $\operatorname{Rect}(\mathbf{x}, \mathbf{y})$ denote the collection of rectangles connecting $\mathbf{x}$ to $\mathbf{y}$. If $\mathbf{x}, \mathbf{y} \in \mathbf{S}$ agree along all but two horizontal circles, then there are exactly two rectangles in $\operatorname{Rect}(\mathbf{x}, \mathbf{y})$; otherwise $\operatorname{Rect}(\mathbf{x}, \mathbf{y})=\varnothing$. A rectangle $r \in \operatorname{Rect}(\mathbf{x}, \mathbf{y})$ is said to be empty if $\operatorname{Int}(r) \cap \mathbf{x}=\varnothing$, or equivalently if $\operatorname{Int}(r) \cap \mathbf{y}=\varnothing$. The space of empty rectangles connecting $\mathbf{x}$ and $\mathbf{y}$ is denoted $\operatorname{Rect}^{\circ}(\mathbf{x}, \mathbf{y})$.

We endow $C^{-}\left(G ; \mathbb{F}_{2}\right)$ with an endomorphism $\partial^{-}: C^{-}\left(G ; \mathbb{F}_{2}\right) \rightarrow C^{-}\left(G ; \mathbb{F}_{2}\right)$ defined by

$$
\partial^{-}(\mathbf{x})=\sum_{\mathbf{y} \in \mathbf{S}} \sum_{r \in \operatorname{Rect}^{\circ}(\mathbf{x}, \mathbf{y})} U_{1}^{O_{1}(r)} \cdots U_{n}^{O_{n}(r)} \cdot \mathbf{y},
$$

where $O_{i}(r)$ denotes the number of times $O_{i}$ appears in the interior of $r$. This differential decreases the Maslov grading by 1 and preserves a filtration by the Alexander grading.

The filtered chain homotopy type of the above complex is a knot invariant [9]; indeed, it is the filtered Heegaard Floer complex $[15 ; 21]$. In the sequel, we will need a slightly less refined version of this. Let $C K^{-}\left(G ; \mathbb{F}_{2}\right)$ be the associated graded object. That is, $C K^{-}\left(G ; \mathbb{F}_{2}\right)$ has the same bigraded set of generators as $C^{-}\left(G ; \mathbb{F}_{2}\right)$, but its differential now counts only empty rectangles with no elements of $\mathbb{X}$ in them; ie, it is given by

$$
\partial(\mathbf{x})=\sum_{\mathbf{y} \in \mathbf{S}} \sum_{\substack{r \in \operatorname{Rect}{ }^{\circ}(\mathbf{x}, \mathbf{y}) \\ r \cap \mathbb{X}=\varnothing}} U_{1}^{O_{1}(r)} \cdots U_{n}^{O_{n}(r)} \cdot \mathbf{y} .
$$


This complex is now bigraded, splitting as $C K^{-}\left(K ; \mathbb{F}_{2}\right)=\bigoplus_{m, a \in \mathbb{Z}} C K_{m}^{-}\left(K, a ; \mathbb{F}_{2}\right)$, so that the differential drops the Maslov grading $m$ by one and preserves the Alexander grading $a$. Thus, its homology groups inherit a bigrading

$$
H F K^{-}\left(K ; \mathbb{F}_{2}\right)=\bigoplus_{m, a \in \mathbb{Z}} H F K_{m}^{-}\left(K, a ; \mathbb{F}_{2}\right)
$$

These groups can be viewed as a bigraded module over $\mathbb{F}_{2}[U]$, where $U$ acts by multiplication by $U_{i}$ for any $i=1, \ldots, n$.

Theorem 3.1 [9] The bigraded homology groups $\operatorname{HFK}^{-}\left(K ; \mathbb{F}_{2}\right)$ agree with the knot Floer homology of $K$ with coefficients in $\mathbb{F}_{2}$.

Moreover, the chain complex can be lifted to $\mathbb{Z}$ coefficients. Let $C^{-}(G)$ be the free $\mathbb{Z}\left[U_{1}, \ldots, U_{n}\right]$ module generated by $\mathbf{S}$. Then we have:

Theorem 3.2 [10, Theorem 4.2] There is an essentially unique function $\mathcal{S}$ from $\operatorname{Rect}(\mathbf{x}, \mathbf{y})$ to $\{ \pm 1\}$ with the following two properties:

- the endomorphism $\partial^{-}: C^{-}(G) \rightarrow C^{-}(G)$ defined by

$$
\partial_{\mathcal{S}}^{-}(\mathbf{x})=\sum_{\mathbf{y} \in \mathbf{S}} \sum_{r \in \operatorname{Rect}^{\circ}(\mathbf{x}, \mathbf{y})} \mathcal{S}(r) \cdot U_{1}^{O_{1}(r)} \cdots U_{n}^{O_{n}(r)} \cdot \mathbf{y}
$$

is a differential; and

- the homology of the associated complex when we set all $U_{i}=1$ has nonzero rank; ie, $H_{*}\left(C^{-}(G) /\left\{U_{i}=1\right\}_{i=1}^{n}\right) \otimes \mathbb{Q}$ is nonzero.

Moreover, the filtered quasi-isomorphism type of the complex $\left(C^{-}(G), \partial_{\mathcal{S}}^{-}\right)$, thought of as a complex over $\mathbb{Z}[U]$ (where $U$ acts as multiplication by any $U_{i}$ ), is an invariant of the link. In particular, it is independent of the choice of $\mathcal{S}$ with the above properties and the grid diagram for $K$.

The proof of Theorem 3.2 gives an independent (and elementary) proof that the homology groups are a topological invariant of $K$.

Remark 3.3 The group $\mathrm{HFK}^{-}(K)$ is the homology group of the associated graded object of the filtered complex $C^{-}(K)$. The filtered quasi-isomorphism type of $C^{-}(K)$ is a more refined knot invariant, and indeed the more general version of Theorem 3.1 identifies this filtered quasi-isomorphism type with a corresponding more general object associated to knots defined using holomorphic disks. In more concrete terms, 
as a result of this extra structure, $\operatorname{HFK}^{-}(K)$ is endowed with a collection of higher differentials, the first of which is

$$
\delta_{1}: \operatorname{HFK}_{d}^{-}(K, s) \rightarrow \operatorname{HFK}_{d-1}^{-}(K, s-1) .
$$

The identification of, say, Theorem 1.1 induce isomorphisms

$$
\phi: H F K_{d}^{-}(K(G), s) \rightarrow H F K_{d}^{-}\left(K\left(G^{\prime}\right), s\right)
$$

which commute with $\delta_{1}$. We have no need for this extra structure in the current paper, but in later work with $\mathrm{Ng}$ we use it to distinguish distinct transverse knots [13, Section 3.2]. (We encounter the filtered chain homotopy type briefly in Appendix A, when comparing the usual definition of $\tau$ with the one discussed in the introduction.)

\section{Grid diagrams and Legendrian knots}

Grid presentations $G$ can represent Legendrian or transverse knots in addition to ordinary knots. Specifically, given a grid presentation $G$ of $K$, we can construct a front projection for a Legendrian realization of the mirror $m(K)$ of $K$ as follows. Consider the projection of $K$ obtained from $G$ as in the previous section. It is a projection with corner points, and indeed there are four types of corner points, which we denote northwest, southwest, southeast and northeast. Smooth all the northwest and southeast corners of the projection, view the southwest and northeast corners as cusps, and then tilt the diagram $45^{\circ}$ clockwise, so that the NE (respectively SW) corners become right (respectively left) cusps. This gives a Legendrian front projection for the mirror of the knot $K$ described by $G$. (It is easy to find a different convention which does not give a mirror; the present conventions appear to fit neatly with conventions on the contact element [16].)

Before giving the combinatorial presentation of Legendrian or transverse knots, we first recall the combinatorial presentation of links using grid diagrams. There are several elementary moves on a grid diagram $G$ that do not change the topological link type:

- (Cyclic permutation) Cyclically permute the rows or columns of $G$.

- (Commutation) For any pair of consecutive columns of $G$ so that the $X$ and $O$ from one column do not separate the $X$ and $O$ on the other column, switch the decorations of these two columns, as in Figure 6. In particular, if the $O$ and $X$ in one column are in adjacent rows, this move can be applied unless there is an $X$ or an $O$ in one of the same rows in the adjacent column. There is also a similar move where the roles of columns and rows are interchanged. 
- (Destabilization) For a corner $c$ which is shared by a pair of vertically-stacked squares $X_{1}, O_{1}$ marked with an $X$ and $O$ respectively, we remove the markings of $X_{1}$ and $O_{1}$ and delete the horizontal and vertical circles containing $c$. Indeed, we can (and do) assume that one of $X_{1}$ or $O_{1}$ meets an additional square marked by an $O$ or an $X$, by the comment above about commutation when there is an adjacent $X$ and $O$. We also can (and do) assume that $c$ is either the lower-left or upper-right corner of $O_{1}$.

- (Stabilization) The inverse of destabilization.

Proposition 4.1 (Cromwell [2], see also Dynnikov [3]) Two grid diagrams represent the same topological link if and only if they can be connected by a sequence of cyclic permutation, commutation, stabilization, and destabilization moves.

We can further classify (de)stabilization moves according to the local configuration of $X$ 's and $O$ 's. Recall that we are assuming now that three marked squares in the original diagram share one corner. There are two data to keep track of: the marking shared by two of these three squares (ie, an $X$ or an $O$ ), and the placement of the unmarked square relative to the shared corner, either $N W, S W, S E$, or $N E$. See Figure 2.
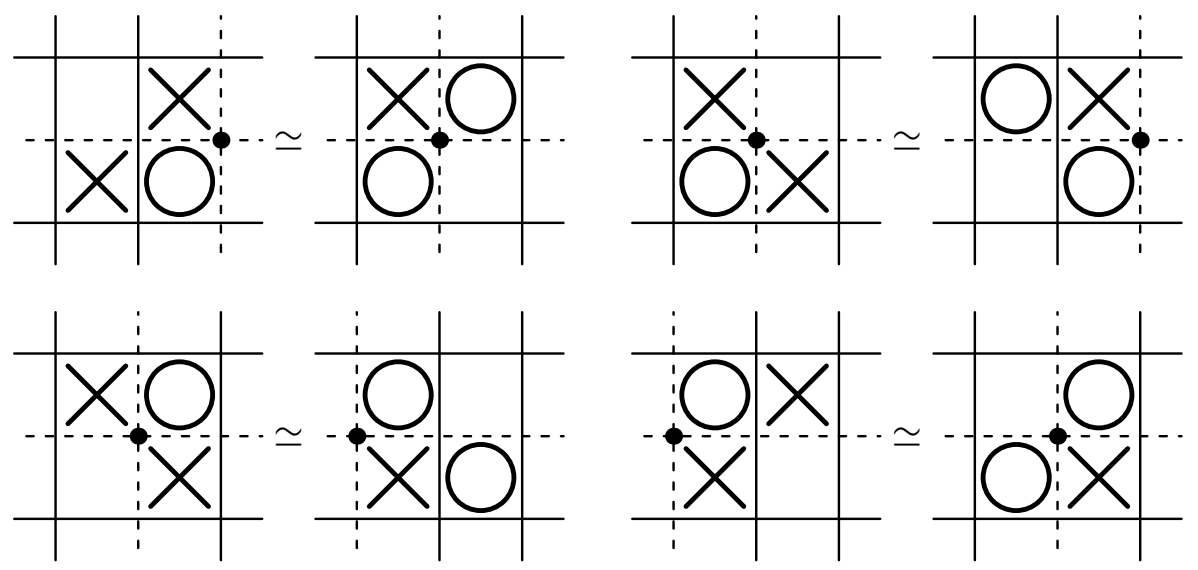

Figure 2: Destabilizations. We have enumerated here the eight types of destabilizations. The dotted lines are to be removed in the destabilized picture. Starting from the upper left corner and reading along the top row first, we have destabilizations of types: $X: N W, O: S E, X: N E, O: S W, X: S W$, $O: N E, X: S E$, and $O: N W$. In each destabilization the two dotted lines are removed and the corner $c$ is marked. The indicated pairs of destabilizations are equivalent modulo commutation moves on the torus. 
The following two lemmas follow by elementary manipulations.

Lemma 4.2 A stabilization of type O:SE (respectively O:NE, O:NW, or O:SW) is equivalent to a stabilization of type $X: N W$ (respectively $X: S W, X: S E$, or $X: N E$ ) followed by a sequence of commutation moves on the torus.

Proof After a stabilization at an $X$ vertex, we can slide either of the resulting segments of length 1 to a neighboring vertex (of type $O$ ) by a sequence of commutation moves. A straightforward check shows that we get a stabilization of the type as indicated in the statement.

Lemma 4.3 A cyclic permutation is equivalent to a sequence of commutations in the plane and (de)stabilizations of types $X: N W, X: S E, O: N W$, and $O: S E$.

Proof Since the allowed moves are symmetric under reversing orientation, we may suppose without loss of generality that we wish to move a horizontal segment from the top to the bottom, with left end of the segment marked $X_{1}$ and right end marked $\mathrm{O}_{2}$. Let $O_{1}$ (respectively $X_{2}$ ) be the other mark in the column containing $X_{1}$ (respectively $\mathrm{O}_{2}$ ). Apply a stabilization of type $X: N W$ at $X_{2}$, and commute the resulting horizontal segment of length 1 to the bottom of the diagram. We now have a vertical segment stretching the height of the diagram; apply commutation moves until it is just to the left of the column containing $X_{1}$. Now the horizontal segment starting at $X_{1}$ is of length 1 , and so can be commuted down until it is just above $O_{1}$, where we can apply a destabilization of type $O: S E$ to get the desired cyclic permutation.

Proposition 4.4 Two grid diagrams represent the same Legendrian link if and only if they can be connected by a sequence of commutation and (de)stabilizations of types $X: N W$ and $X: S E$ on the torus.

Proof By Lemmas 4.2 and 4.3, we can equivalently consider commutation and (de)stabilizations of all types $N W$ and $S E$ in the rectangle (rather than on the torus).

We must now check that each type of commutation and allowed stabilization (in the rectangle) gives an isotopy of the corresponding Legendrian knot. Indeed, after rotating $45^{\circ}$ and turning the corners into smooth turns or cusps as appropriate, each elementary move of the grid diagram becomes a sequence of Legendrian Reidemeister moves of the front projection. For instance, as shown in Figure 3, an $S E$ stabilization becomes either a planar isotopy or a Legendrian Reidemeister 1 move, depending on the relation of the stabilized corner to the rest of the diagram. An example of a commutation move that 


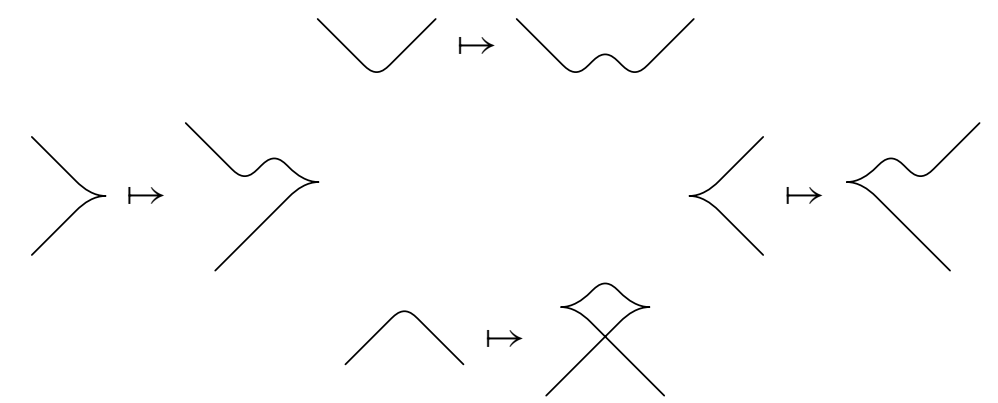

Figure 3: The four different ways an $S E$ stabilization can appear after converting into a Legendrian front. Three of them are planar isotopy, while the fourth is an allowed Reidemeister move on Legendrian knots.

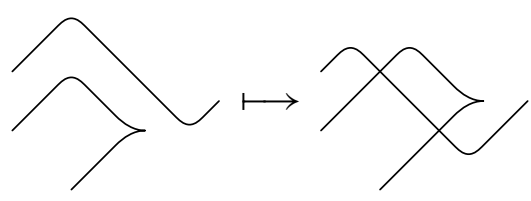

Figure 4: A commutation move giving a Legendrian Reidemeister 2 move.

turns into a Legendrian Reidemeister 2 move is shown in Figure 4; other commutation moves are similar, and may also involve Reidemeister 3 moves.

To go the other direction, we must show that every sequence of Reidemeister moves on a Legendrian front can be turned into a corresponding sequence of grid moves. First note that any Legendrian front projection can be turned into a grid diagram: Take the Legendrian front and stretch it horizontally until no portion of the diagram is at an angle of more than $45^{\circ}$ from the horizontal. Then the curve can be approximated by a sequence of straight segments at an angle of $\pm 45^{\circ}$. After rotating by $45^{\circ}$ counterclockwise and adjusting the segments to have consecutive integer coordinates, we have a grid diagram corresponding to the front projection.

We can do the same thing with any Legendrian isotopy: stretch the intermediate diagrams so that no edges are too steep and approximate each one by a sequence of straight segments. It is an elementary verification that each modification along the way (ie, change of the approximation by segments and Legendrian Reidemeister moves) can be achieved by a sequence of commutation and allowed destabilizations.

Furthermore, a stabilization of type $X: S W$ is a negative stabilization of the Legendrian link (see Figure 5), so we have: 


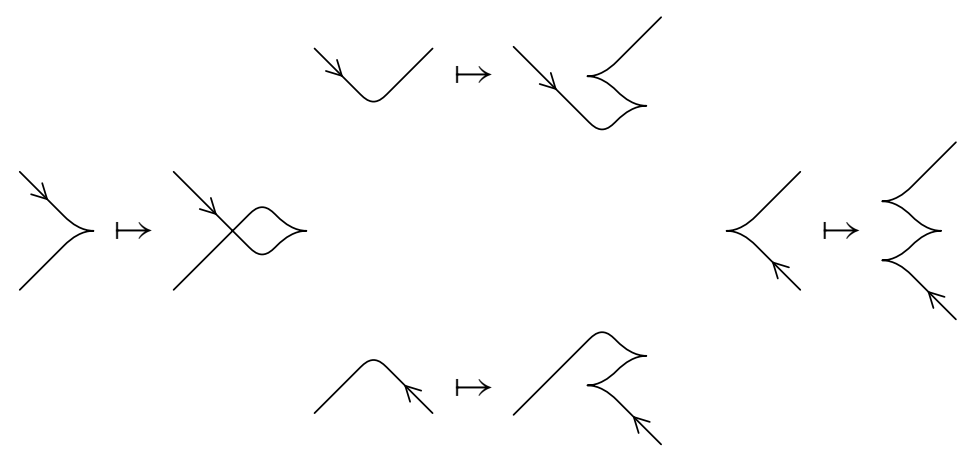

Figure 5: Stabilizations of type $X: S W$, after conversion to a Legendrian front. In each case the Legendrian knot type is changed by a negative stabilization.

Corollary 4.5 Two grid diagrams represent the same transverse link if and only if they can be connected by a sequence of commutation and (de)stabilizations of types $X: N W$, $X: S E$, and $X: S W$.

Finally we note how symmetries of the grid diagram relate to symmetries of the knot. We consider only the symmetries that preserve the set of cusps.

Lemma 4.6 Symmetries of a grid diagram $G$ take an oriented Legendrian knot $\overrightarrow{\mathcal{K}}$ to the following Legendrian knots:

- Reflection through the $x=-y$ axis: $-\overrightarrow{\mathcal{K}}$;

- Reflection through the $x=y$ axis: $-\mu(\overrightarrow{\mathcal{K}})$; and

- Rotation by $180^{\circ}: \mu(\overrightarrow{\mathcal{K}})$.

Proof Reflection of $G$ through the $x=-y$ axis corresponds to reflecting the front projection through the $z$ (vertical) axis, which in turn corresponds to rotating the Legendrian knot by $180^{\circ}$ around the $z$-axis, which is a Legendrian isotopy. (The fact this is a Legendrian isotopy is most easily seen by using the isotopic contact form $d z-(x d y-y d x) / 2$, which is rotationally symmetric.) Moreover, by considering the orientation conventions for knot diagrams, one can easily see that this operation also reverses the orientation of the knot.

Reflection of $G$ through the $x=y$ axis similarly corresponds to rotation of the Legendrian projection by $180^{\circ}$ around the axis normal to the Legendrian projection (which is the definition of Legendrian mirror), followed by a reflection through the $x=-y$ axis (which does not affect the Legendrian isotopy class). This operation also reverses the orientation.

Rotation of $G$ by $180^{\circ}$ is the composition of the previous two symmetries. 


\section{Grid moves for knot Floer homology}

We recall here the explicit maps induced by commutation and destabilization moves in the combinatorial proof of topological invariance of $\mathrm{HFK}^{-}$[10]. We will give the formulas over $\mathbb{F}_{2}$; signs can be added to all these formulas to give formulas that work over $\mathbb{Z}[10$, Section 4.2].

\subsection{Commutation maps}

More explicitly, suppose that $G$ and $H$ are two grid diagrams for the same oriented knot $\vec{K}$, which differ by commuting two vertical edges. It is convenient to draw both diagrams on the same torus, replacing a distinguished vertical circle $\beta$ for $G$ with a different one $\gamma$ for $H$, as pictured in Figure 6. The circles $\beta$ and $\gamma$ meet each other transversally in two points $a$ and $b$, which are not on a horizontal circle.

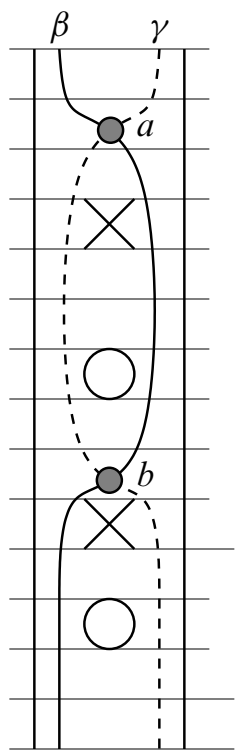

Figure 6: Commutation. A commutation move, viewed as replacing one vertical circle ( $\beta$, undashed) with another $(\gamma$, dashed).

We define a chain map $\Phi_{\beta \gamma}: C^{-}(G) \rightarrow C^{-}(H)$ by counting pentagons in the torus. Given $\mathbf{x} \in \mathbf{S}(G)$ and $\mathbf{y} \in \mathbf{S}(H)$, we let $\operatorname{Pent}_{\beta \gamma}(\mathbf{x}, \mathbf{y})$ denote the space of embedded pentagons with the following properties, as illustrated in Figure 10. This space is empty unless $\mathbf{x}$ and $\mathbf{y}$ coincide at $n-2$ points. An element of Pent $\beta_{\beta \gamma}(\mathbf{x}, \mathbf{y})$ is an embedded disk in $T^{2}$, whose boundary consists of five arcs, each contained in horizontal or 
vertical circles. Moreover, under the orientation induced on the boundary of $p$, we start at the $\beta$-component of $\mathbf{x}$, traverse the arc of a horizontal circle, meet its corresponding component of $\mathbf{y}$, proceed along an arc of a vertical circle, meet the corresponding component of $\mathbf{x}$, continue through another horizontal circle, meet the component of $\mathbf{y}$ contained in the distinguished circle $\gamma$, proceed along an arc in $\gamma$, meet an intersection point of $\beta$ with $\gamma$, and finally, traverse an arc in $\beta$ until we arrive back at the initial component of $\mathbf{x}$. Finally, all the angles here are required to be acute. These conditions imply that there is a particular intersection point, denoted $a$, between $\beta$ and $\gamma$ which appears as one of the corners of any pentagon in $\operatorname{Pent}_{\beta \gamma}(\mathbf{x}, \mathbf{y})$. The other intersection point $b$ appears in all of the pentagons in $\operatorname{Pent}_{\gamma \beta}(\mathbf{y}, \mathbf{x})$. The space of empty pentagons $p \in \operatorname{Pent}_{\beta \gamma}(\mathbf{x}, \mathbf{y})$ with $\mathbf{x} \cap \operatorname{Int}(p)=\varnothing$, is denoted Pent ${ }_{\beta \gamma}^{\circ}$.

Given $\mathbf{x} \in \mathbf{S}(G)$, define

$$
\Phi_{\beta \gamma}(\mathbf{x})=\sum_{\mathbf{y} \in \mathbf{S}(H)} \sum_{p \in \operatorname{Pent}_{\beta \gamma}^{\circ}(\mathbf{x}, \mathbf{y})} U_{1}^{O_{1}(p)} \cdots U_{n}^{O_{n}(p)} \cdot \mathbf{y} \in C^{-}(H) .
$$

It is elementary to see that the above map induces a chain homotopy equivalence [10, Proposition 3.2].

In this paper we will consider the above map on the associated graded object $C K^{-}(K)$, ie, where we count $p \in$ Pent $_{\beta \gamma}^{\circ}$ subject to the further constraint that $p \cap \mathbb{X}=\varnothing$.

\subsection{Stabilization maps}

Next, we consider the stabilization map. Let $G$ be a grid diagram and $H$ denote a stabilization. We discuss in detail the case where we introduce a new column with $O_{1}$ immediately above $X_{1}$, and there is another marking $X_{2}$ immediately to the left or to the right of $O_{1}$, as is the case of two of the four types of $X$-stabilization; the cases where $X_{1}$ is immediately above $O_{1}$ can be treated symmetrically by atation of all diagrams by $180^{\circ}$.

Label the $O$ in the same row as $X_{1}$ by $O_{2}$. Let $\beta_{1}$ be the vertical circle just to the left of $O_{1}$ and $X_{1}$, and let $\alpha$ denote the horizontal circle separating the squares marked $O_{1}$ and $X_{1}$.

Let $B=C^{-}(G), C=C^{-}(H)$, and let $C^{\prime}$ be the mapping cone of

$$
U_{2}-U_{1}: B\left[U_{1}\right] \longrightarrow B\left[U_{1}\right],
$$

ie, $C^{\prime}\left[U_{1}\right]=B\left[U_{1}\right] \oplus B\left[U_{1}\right]$, endowed with the differential $\partial^{\prime}: C^{\prime} \rightarrow C^{\prime}$ given by

$$
\partial^{\prime}(a, b)=\left(\partial^{-} a,\left(U_{2}-U_{1}\right) \cdot a-\partial^{-} b\right)
$$


where here $\partial^{-}$denotes the differential within $B$. Note that $B$ is a chain complex over $\mathbb{Z}\left[U_{2}, \ldots, U_{n}\right]$, so that $B\left[U_{1}\right]$ denotes the induced complex over $\mathbb{Z}\left[U_{1}, \ldots, U_{n}\right]$ gotten by introducing a new formal variable $U_{1}$. Let $\mathcal{L} \cong B\left[U_{1}\right]$ (respectively $\mathcal{R}$ ) be the subgroup of $C^{\prime}$ of elements of the form $(c, 0)$ (respectively $(0, c)$ ) for $c \in B\left[U_{1}\right]$. The module $\mathcal{R}$ inherits Alexander and Maslov gradings from its identification with $B\left[U_{1}\right]$, while $\mathcal{L}$ is given the Alexander and Maslov gradings which are one less than those it inherits from its identification with $B\left[U_{1}\right]$. With respect to these conventions, the mapping cone is a filtered complex of $\mathcal{R}$-modules. We claim that $C^{\prime}$ is quasiisomorphic to $B$. In fact, it is straightforward to verify that a quasi-isomorphism is given by the map $(a, b) \mapsto b^{\prime}$, where here $b^{\prime}$ denotes the element of $B$ gotten by taking $b \in B\left[U_{1}\right]$, and substituting $U_{2}$ for the formal variable $U_{1}$.

Furthermore, there is a filtered quasi-isomorphism

$$
F: C \longrightarrow C^{\prime} .
$$

To describe this, we introduce a little more notation.

Let $x_{0}$ be the intersection point of $\alpha$ and $\beta_{1}$. Let $\mathbf{I} \subset \mathbf{S}(H)$ be the set of $\mathbf{x} \in \mathbf{S}(H)$ which contain $x_{0}$. There is, of course, a natural (point-wise) identification between $\mathbf{S}(G)$ and $\mathbf{I}$, which drops Alexander and Maslov grading by one.

As such, the differentials within $\mathcal{L}$ and $\mathcal{R}$ count rectangles in $H$ which do not contain $x_{0}$ on their boundary, although they may contain $x_{0}$ in their interior. Note however that the boundary operator for rectangles containing $x_{0}$ does not involve the variable $U_{1}$.

For the definition of $F$, we must consider objects more general than rectangles, called domains. To define them, let us view the torus $T^{2}$ as a two-dimensional cell complex, with the toroidal grid diagram inducing the cell decomposition with $n^{2}$ zero-cells, $2 n^{2}$ one-cells and $n^{2}$ two-cells (the little squares). Let $U_{\alpha}$ be the one-dimensional subcomplex of $T^{2}$ consisting of the union of the $n$ horizontal circles.

Definition 5.1 Given $\mathbf{x}, \mathbf{y} \in \mathbf{S}(H)$, a path from $\mathbf{x}$ to $\mathbf{y}$ is a 1-cycle $\gamma$ on the cell complex $T^{2}$, such that the boundary of the intersection of $\gamma$ with $U_{\alpha}$ is $\mathbf{y}-\mathbf{x}$. A domain $p$ from $\mathbf{x}$ to $\mathbf{y}$ is a two-chain in $T^{2}$ whose boundary $\partial p$ is a path from $\mathbf{x}$ to $\mathbf{y}$. The multiplicity of $O_{i}$ in a domain $p$, denoted $O_{i}(p)$, is the local multiplicity of the chain $p$ at $O_{i}$.

For example, if $p$ is a rectangle from $\mathbf{x}$ to $\mathbf{y}$, then the above definition of $O_{i}(r)$ coincides with the earlier definition used in Equation (6).

Only certain domains will be counted in the definition of $F$. 

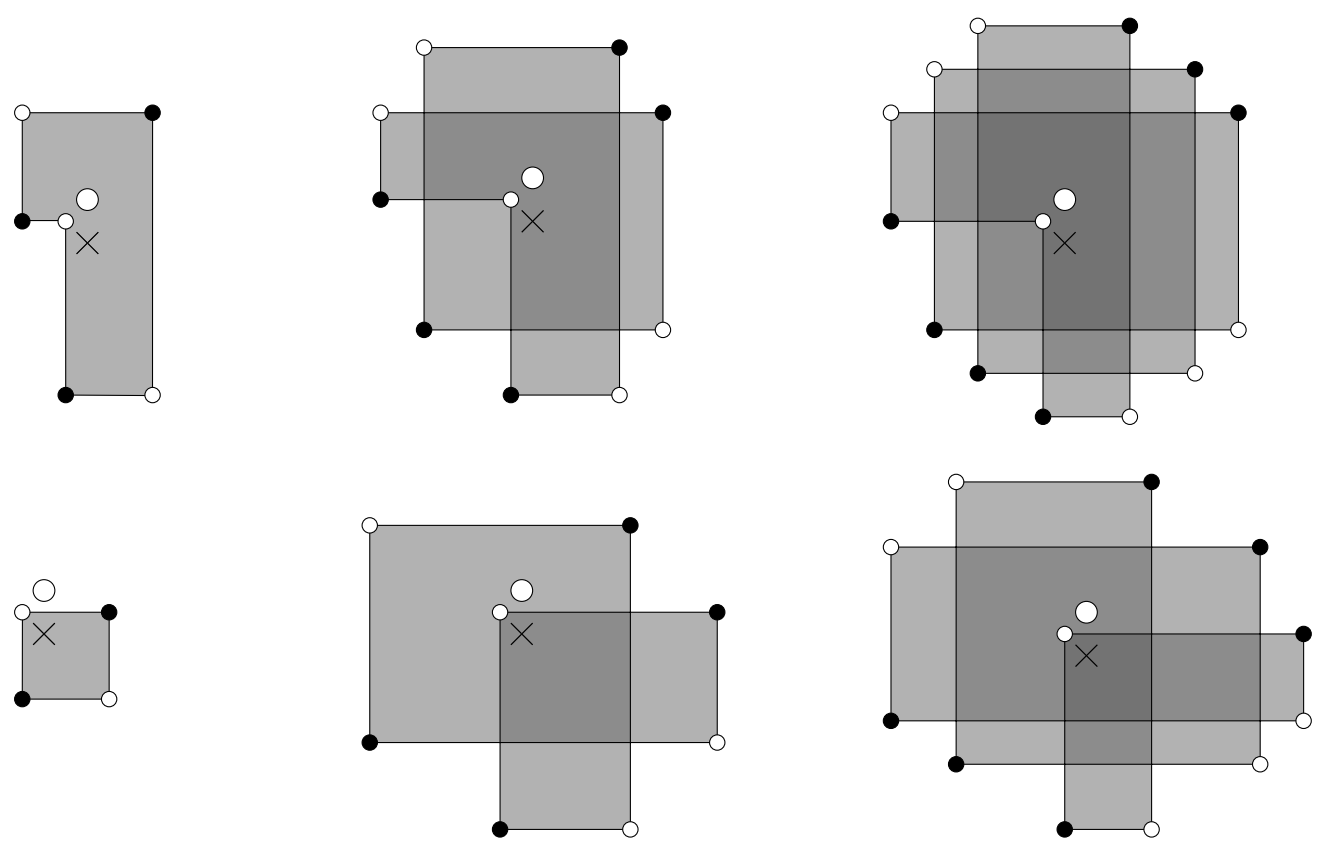

Figure 7: Types of domains. We have listed here several domains in the stabilized diagram, labeling the initial points by dark circles, and terminal points by empty circles. The top row lists domains of type $L$, while the second row lists some of type $R$. The marked $O$ and $X$ are the new ones in the stabilized picture. Darker shading corresponds to higher local multiplicities.

Definition 5.2 For $\mathbf{x} \in \mathbf{S}(H)$ and $\mathbf{y} \in \mathbf{I} \subset \mathbf{S}(H)$, a domain $p \in \pi(\mathbf{x}, \mathbf{y})$ is said to be of type $L$ (respectively $R$ ) if either it is the zero chain, in which case $p$ has type $L$, or it satisfies the following conditions:

- $\quad p$ has only nonnegative local multiplicities.

- For each $c \in \mathbf{x} \cup \mathbf{y}$, other than $x_{0}$, at least three of the four adjoining squares have vanishing local multiplicities.

- In a neighborhood of $x_{0}$ the local multiplicities in three of the adjoining rectangles are the same number $k$. When $p$ has type $L$, the lower left corner has local multiplicity $k-1$, while for $p$ of type $R$ the lower right corner has multiplicity $k+1$.

- $\partial p$ is connected. 
The set of type $L$ (respectively $R$ ) domains from $\mathbf{x}$ to $\mathbf{y}$ is denoted $\pi^{L}(\mathbf{x}, \mathbf{y})$ (respectively $\left.\pi^{R}(\mathbf{x}, \mathbf{y})\right)$. See Figure 7 for examples.

We now define maps

$$
\begin{aligned}
& F^{L}: C \longrightarrow \mathcal{L} \\
& F^{R}: C \longrightarrow \mathcal{R}
\end{aligned}
$$

where $F^{L}$ (respectively $F^{R}$ ) counts domains of type $L$ (respectively $R$ ) without factors of $U_{1}$. Specifically, define

$$
\begin{aligned}
& F^{L}(\mathbf{x})=\sum_{\mathbf{y} \in \mathbf{I}} \sum_{p \in \pi^{L}(\mathbf{x}, \mathbf{y})} U_{2}^{O_{2}(p)} \cdots U_{n}^{O_{n}(p)} \cdot \mathbf{y} \\
& F^{R}(\mathbf{x})=\sum_{\mathbf{y} \in \mathbf{I}} \sum_{p \in \pi^{R}(\mathbf{x}, \mathbf{y})} U_{2}^{O_{2}(p)} \cdots U_{n}^{O_{n}(p)} \cdot \mathbf{y} .
\end{aligned}
$$

In order to identify the range of $F^{L}$ (respectively $F^{R}$ ) with $\mathcal{L}$ (respectively $\mathcal{R}$ ), we implicitly use the identification $\mathbf{S}(G) \cong \mathbf{I} \subset \mathbf{S}(H)$.

We put these together to define a map

$$
F=\left(\begin{array}{l}
F^{L} \\
F^{R}
\end{array}\right): C \longrightarrow C^{\prime}
$$

The fact that $F$ is a quasi-isomorphism is established in [10, Proposition 3.8].

Again, $F$ induces also a quasi-isomorphism on the associated graded object, giving a map from $C K^{-}(H)$ to the mapping cone of multiplication by $U_{1}-U_{2}$, thought of as an endomorphism of $C K^{-}(G)\left[U_{1}\right]$. This induced map counts only those domains $p$ of type $F$ for which $X_{i}(p)=0$ for all $i=2, \ldots, n$. (Note that we do allow $X_{1}(p) \neq 0$.)

\section{Definition and invariance properties of the Legendrian in- variants}

We this setup, we can now construct the Legendrian invariant for knots. The case of links works with minor modifications, as spelled out in Section 7.

Definition 6.1 Let $G$ be a grid diagram for a knot, and consider the chain complex $C K^{-}(G)$. Consider elements $\boldsymbol{x}^{+}, \boldsymbol{x}^{-} \in \mathbf{S}(G)$ defined as follows. Each component of $\boldsymbol{x}^{+}$is the upper right corner of some square decorated with $X$, while each component of $\boldsymbol{x}^{-}$is the lower left corner of some square decorated with $X$. The chains $z^{+}$and $z^{-}$are defined to be $\pm x^{+}$and $\pm x^{-}$, respectively, with signs specified in Definition 6.10 . 
We will defer most discussion of signs until later. For now, all the proofs will work with an arbitrary choice of signs, although at present we will only prove some of the results up to a choice of sign. We now verify that the above chains are in fact cycles.

Lemma 6.2 The elements $z^{+}$and $z^{-}$are cycles in the chain complex $C K^{-}(G)$.

Proof Consider any $y \in \mathbf{S}(G)$ and $r \in \operatorname{Rect}\left(x^{+}, \mathbf{y}\right)$. Let $x_{1} \in \boldsymbol{x}^{+}$be the upper right corner of $r$. By the definition of $\boldsymbol{x}^{+}$, there is an $X$ in the square to the lower left of $x_{1}$, and hence also an $X$ in $r$. Thus, $r$ cannot count in the definition of the differential $\partial$ for the associated graded object. An analogous argument applies to $x^{-}$.

Next, we calculate the Maslov and Alexander gradings.

Lemma 6.3 We have that $A\left(x^{+}\right)=\frac{1}{2} M\left(x^{+}\right)$and $A\left(x^{-}\right)=\frac{1}{2} M\left(x^{-}\right)$.

Proof Recall that $A\left(x^{+}\right)=\frac{1}{2}\left(M_{\mathbb{O}}\left(x^{+}\right)-M_{\mathbb{X}}\left(x^{+}\right)\right)-(n-1 / 2)$. Because of the close relationship of $\boldsymbol{x}^{+}$and $\mathbb{X}$, we have some equalities on the terms appearing in $M_{\mathbb{X}}$ when we work in a fundamental domain including the right and top edges:

$$
\begin{aligned}
\mathcal{I}\left(\boldsymbol{x}^{+}, \boldsymbol{x}^{+}\right) & =\mathcal{I}(\mathbb{X}, \mathbb{X})=\mathcal{I}\left(\boldsymbol{x}^{+}, \mathbb{X}\right) \\
\mathcal{I}\left(\mathbb{X}, \boldsymbol{x}^{+}\right) & =\mathcal{I}(\mathbb{X}, \mathbb{X})+n
\end{aligned}
$$

Therefore $M_{\mathbb{X}}\left(\boldsymbol{x}^{+}\right)=-n+1$ and $A\left(\boldsymbol{x}^{+}\right)=\frac{1}{2} M_{\mathbb{O}}\left(\boldsymbol{x}^{+}\right)$as desired. A similar argument applies to $\boldsymbol{x}^{-}$.

Let $K$ be the knot diagram represented by the grid diagram. Remember that the topological type of $K$ is the mirror of the topological type of $\overrightarrow{\mathcal{K}}$.

Lemma 6.4 The Maslov gradings of the elements $\boldsymbol{x}^{+}$and $\boldsymbol{x}^{-}$are given by

$$
\begin{aligned}
& M\left(\boldsymbol{x}^{+}\right)=-\operatorname{wr}(K)-\#\{\text { downward-oriented cusps }\}+1 \\
& M\left(\boldsymbol{x}^{-}\right)=-\operatorname{wr}(K)-\#\{\text { upward-oriented cusps }\}+1 .
\end{aligned}
$$

Proof Each horizontal segment $K_{i}$ of $K$ goes from some $O_{i}$ to some $X_{i}$. Let $x_{i}$ be the point in $\boldsymbol{x}^{+}$to the upper right of $X_{i}$. We claim that the quantity $C_{i}$ defined by

$$
C_{i}:=\mathcal{J}\left(\left\{x_{i}\right\}-\left\{O_{i}\right\}, \boldsymbol{x}^{+}-\mathbb{O}\right)
$$

is given by

$$
\begin{aligned}
C_{i}= & \#\left\{\text { negative crossings on } K_{i}\right\}-\#\left\{\text { positive crossings on } K_{i}\right\} \\
& -\#\left\{\text { downward-oriented cusps among }\left\{X_{i}, O_{i}\right\}\right\} .
\end{aligned}
$$


To prove this, we use the horizontal segment to divide up the plane into four regions, as follows. Let $A$ be the vertical column through $X_{i}, B$ be the vertical column through $O_{i}$, $C$ be the vertical strip between $A$ and $B$ (note that the region $C$ can be empty when the arc from $O_{i}$ to $X_{i}$ has length one), and $D$ be the complement of $A \cup B \cup C$. (Note that $D$ generally has two connected components.) Each region should be interpreted as including its right boundary. Then for each $O_{j}$ in $D, \mathcal{J}\left(\left\{x_{i}\right\},\left\{O_{j}\right\}\right)=\mathcal{J}\left(\left\{O_{i}\right\},\left\{O_{j}\right\}\right)$, and similarly for each $x_{j}$ in $D, \mathcal{J}\left(\left\{x_{i}\right\},\left\{x_{j}\right\}\right)=\mathcal{J}\left(\left\{O_{i}\right\},\left\{x_{j}\right\}\right)$. Thus

$$
\mathcal{J}\left(\left\{x_{i}\right\}-\left\{O_{i}\right\},\left(x^{+}-\mathbb{O}\right) \cap D\right)=0 .
$$

Next consider $O_{j} \in C$, so that its corresponding $X_{k}$ which lies in the same column also is in $C$. If the vertical arc connecting $O_{j}$ to $X_{k}$ does not cross $K_{i}$, we have that

and

$$
\begin{aligned}
& \mathcal{J}\left(\left\{x_{i}\right\},\left\{O_{j}\right\}\right)=\mathcal{J}\left(\left\{x_{i}\right\},\left\{x_{k}\right\}\right) \\
& \mathcal{J}\left(\left\{O_{i}\right\},\left\{O_{j}\right\}\right)=\mathcal{J}\left(\left\{O_{i}\right\},\left\{x_{k}\right\}\right) .
\end{aligned}
$$

Otherwise both equalities are off by $\pm 1 / 2$. A more careful look at the orientation of the horizontal and vertical arcs and our conventions on the crossing type reveals that in fact

$$
\begin{aligned}
\mathcal{J}\left(\left\{x_{i}\right\}-\left\{O_{i}\right\},\left(x^{+}-\mathbb{O}\right) \cap C\right)= & \#\left\{\text { negative crossings on } K_{i}\right\} \\
& -\#\left\{\text { positive crossings on } K_{i}\right\} .
\end{aligned}
$$

Finally, we claim that

$$
\mathcal{J}\left(\left\{x_{i}\right\}-\left\{O_{i}\right\},\left(x^{+}-\mathbb{O}\right) \cap(A \cup B)\right)=-\#\left\{\text { downward cusps among }\left\{X_{i}, O_{i}\right\}\right\} .
$$

This follows from an analysis of the eight cases: whether the $O_{j} \in A$ is above or below $X_{i}$, whether the $X_{k} \in B$ is above or below $O_{i}$, and whether $O_{i}$ is to the left or to the right of $X_{i}$. These eight cases are illustrated in Figure 8.

Equation (11) now follows by adding up Equations (14), (13), and (12). Equation (9) follows from Equation (11) by adding up the contributions of each horizontal arc, and then adding one (as in Equation (4)). Equation (10) follows from a similar analysis, except that in this case, the contribution from the regions in $A$ and $B$ are different, and so we replace Equation (14) by the following:

$$
\mathcal{J}\left(\left\{x_{i}\right\}-\left\{O_{i}\right\},\left(x^{-}-\mathbb{O}\right) \cap(A \cup B)\right)=-\#\left\{\text { upward cusps among }\left\{X_{i}, O_{i}\right\}\right\} .
$$

Lemma 6.5 Let $G$ be a grid diagram and $H$ a stabilization of $G$ of type $X: N W$ or $X: S E$. Then the destabilization map from $C^{-}(H)$ to the mapping cone of

$$
U_{1}-U_{2}: C^{-}(G)\left[U_{1}\right] \longrightarrow C^{-}(G)\left[U_{1}\right]
$$



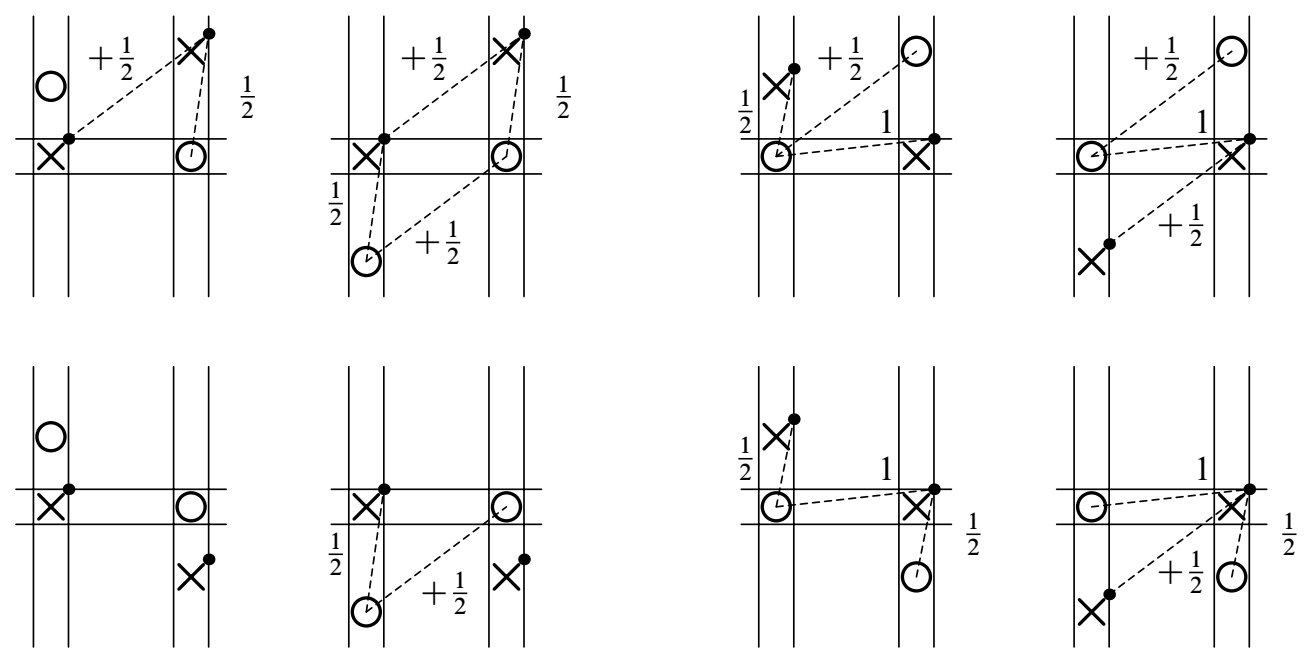

Figure 8: By considering the eight illustrated possibilities, we verify Equation (14). The relevant contributions to $C_{i}$ are indicated by dashed lines, labelled by the value of the contribution. In each case the sum of the contributions equals the number of downward-oriented cusps in the front projection.

carries the two elements $z^{+}(H)$ and $z^{-}(H)$ to $\pm z^{+}(G)$ and $\pm z^{-}(G)$, thought of as an element of $C^{-}(G) \subset \mathcal{R} \subset C^{\prime}$, in the notation of Section 5.2 (ie, where the three markings involved are $X_{1}, O_{1}$, and $X_{2}$ ).

Proof This follows from a case analysis of the stabilizations.

Let $\boldsymbol{x}^{+\prime}$ be the canonical generator for the stabilized diagram, and $\boldsymbol{x}^{+}$the corresponding generator in the destabilized diagram, considered as an element of $\mathcal{R}$.

In each case, we claim that there is only one nonzero term in $F\left(x^{+\prime}\right)$, since there is exactly one domain in $\pi^{F}\left(x^{+\prime}, \mathbf{y}\right)$ which does not contain any of the $X_{i}$ with $i \neq 1$. As in Lemma 6.2, this follows by considering the upper right corner of any such domain, which must be $X_{1}$. Moreover, we claim that for this domain, the image point $\mathbf{y}$ corresponds to the canonical generator $\boldsymbol{x}^{+}$for the destabilized picture. We have illustrated the two cases on the left of Figure 9. Because there is only one domain, the destabilization map carries $z^{+}(H)$ to $\pm z^{+}(G)$ as desired. As sketched on the right of Figure 9, a similar argument works for $\boldsymbol{x}^{-}$.

Lemma 6.6 Let $G$ and $H$ be two grid diagrams which differ by a commutation move. Then under the map $F_{\beta \gamma}: C^{-}(G) \rightarrow C^{-}(H)$, the image of the cycle $z^{+}(G)$ is $\pm z^{+}(H)$ and the image of $z^{-}(G)$ is $\pm z^{-}(H)$. 

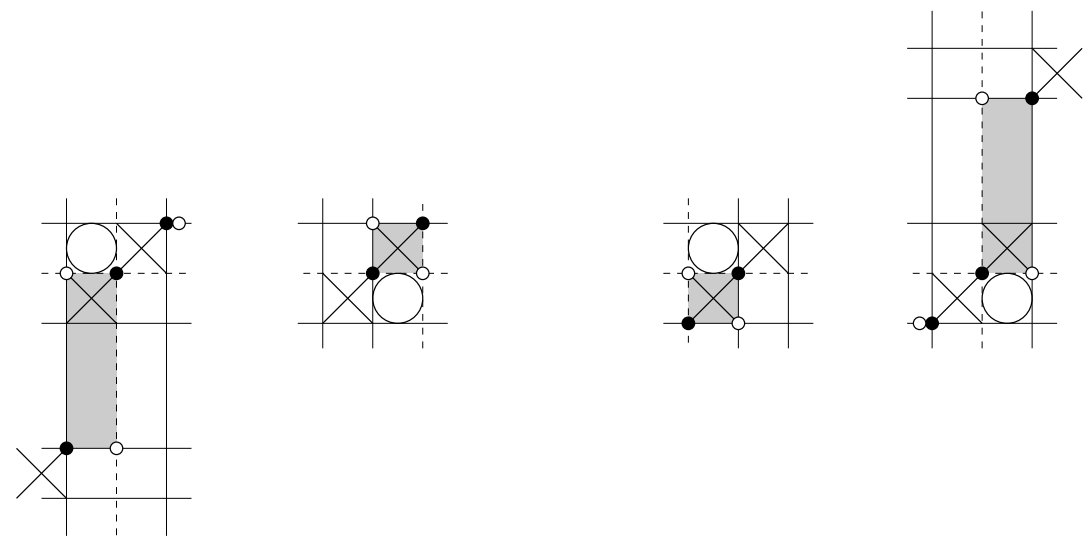

Figure 9: Invariance of the Legendrian element. We have illustrated a local picture of a stabilized diagram. The black dots represent the canonical elements for the stabilized diagram, the white ones represent the elements afterwards, ie, they are destabilized at the pair of dotted lines. The left two diagrams show $\boldsymbol{x}^{+\prime}$, while the right two show $\boldsymbol{x}^{-\prime}$. In all four cases the destabilization map counts the shaded rectangle, a region with complexity 2 of type $R$.

Proof We argue that there is exactly one $\mathbf{y}$ and one pentagon in $\operatorname{Pent}\left(\boldsymbol{x}^{+}(G), \mathbf{y}\right)$ which does not contain some $X_{i}$ in its interior, and that is the one which connects $\boldsymbol{x}^{+}(G)$ to $\boldsymbol{x}^{+}(H)$. This can be seen from an argument like that in Lemma 6.5: there is a pentagon taking $\boldsymbol{x}^{+}(G)$ to $\boldsymbol{x}^{+}(H)$, as illustrated in Figure 10. Suppose that $\psi$ is any other pentagon, and consider its upper right corner, which is at some $c \in \boldsymbol{x}^{+}(G)$. The subsquare just to the lower left of this $c$ contains some $X_{i}$ with $i \neq 1$ or 2 . The argument for $\boldsymbol{x}^{-}$is similar.

Proof of Theorem 1.1, up to signs The calculations of the Maslov and Alexander gradings of $z^{+}$are Lemmas 6.4 and 6.3 respectively. The Alexander grading is given by

$$
\begin{aligned}
A\left(z^{+}\right) & =-\operatorname{wr}(K)-\#\{\text { downward-oriented cusps }\} \\
& =\operatorname{wr}(m(K))-\#\{\text { downward-oriented cusps\}, } \\
A\left(z^{-}\right) & =\operatorname{wr}(m(K))-\#\{\text { upward-oriented cusps\}. }
\end{aligned}
$$

Comparing these with the standard descriptions of tb and $r$ from the knot projection (Equations (2) and (3) respectively), we obtained the stated formulas for the bigradings of $z^{+}$and $z^{-}$. 


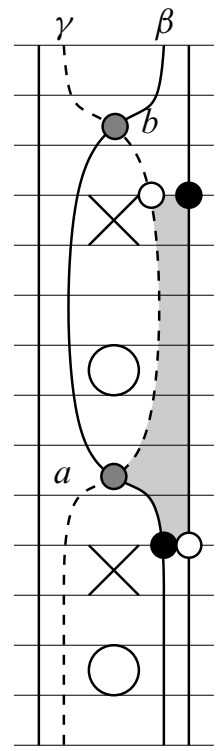

Figure 10: Small pentagon. The dark circles represent the canonical generator $\boldsymbol{x}^{+}$for $G$, the diagram involving $\beta$, while the empty circles represent the canonical generator $\boldsymbol{x}^{+\prime}$ for $H$, the diagram involving $\gamma$. The shaded pentagon represents the map carrying $x^{+}$to $x^{+\prime}$.

In view of Proposition 4.4, invariance under Legendrian isotopies (up to sign) follows from Lemmas 6.5 and 6.6.

\subsection{Properties of the Legendrian invariant}

We now turn to the properties of $\lambda^{+}$and $\lambda^{-}$stated in the introduction.

Proof of Proposition 1.2, up to signs The fact that the two invariants are permuted under orientation reversal follows from the symmetry of the torus which is given by reflection through the $x=-y$ axis. More precisely, it is easy to see that if $G$ is a grid diagram representing a Legendrian knot $\overrightarrow{\mathcal{K}}$ and $H$ is the grid diagram obtained from $G$ by reflecting through this axis, then the reflection map from $\mathbf{S}(G)$ to $\mathbf{S}(H)$, which takes $\boldsymbol{x}^{+}(G)$ to $\boldsymbol{x}^{-}(H)$ and $\boldsymbol{x}^{-}(G)$ to $\boldsymbol{x}^{+}(H)$, induces an isomorphism of complexes

$$
\Phi: C^{-}(G) \longrightarrow C^{-}(H),
$$

with $\Phi\left(z^{+}(G)\right)= \pm z^{-}(H)$ and $\Phi\left(z^{-}(G)\right)= \pm z^{+}(H)$. Moreover, by Lemma 4.6, the Legendrian knot specified by $H$ is $-\overrightarrow{\mathcal{K}}$. 
Similarly, rotation of a grid diagram $G$ by $180^{\circ}$ induces an isomorphism of chain complexes which permutes the two canonical cycles while taking $\overrightarrow{\mathcal{K}}$ to its Legendrian mirror $\mu(\overrightarrow{\mathcal{K}})$.

Proof of Theorem 1.3, up to signs. Behavior under positive and negative destabilization is illustrated in Figure 11. The illustrated domains, all of complexity 2 and type $R$, are as before the only domains of type $F$ starting at $\boldsymbol{x}^{+}$or $\boldsymbol{x}^{-}$which do not contain any other $X_{i}$ in their interior. In all cases the rectangle connects $\mathbf{x}^{ \pm}$to the canonical generator for the destabilized picture. For the positive destabilization of $\boldsymbol{x}^{+}$ and the negative destabilization of $\boldsymbol{x}^{-}$, the domain contains the other $O$ in the row containing $X_{1}$ (and so the map on homology is multiplication by $U$ ), while for the other two cases the domain contains only $X_{1}$. Therefore the induced map $\Phi$ on the chain complex behaves as stated, up to a sign.
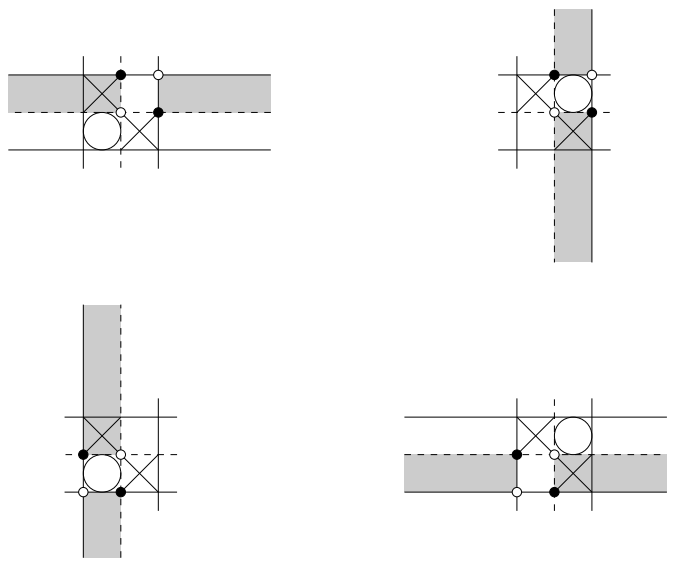

Figure 11: Behavior under positive and negative destabilization. The black generators represent the canonical elements for the stabilized diagram, while the white ones represent canonical elements in the destabilized one. The left two diagrams are positive destabilization (of type $X: N E$ ) and the right two are negative destabilization (of type $X: S W$ ). On the top row, we consider $\boldsymbol{x}^{+}$, while on the bottom row, we consider $\boldsymbol{x}^{-}$.

In order to prove the invariant is nonzero (Theorem 1.5), we will look at yet another complex.

Definition 6.7 Let the complex $C^{!}(G)$ be the tensor product of $C K^{-}(G)$ with Laurent polynomials in the $\left\{U_{i}\right\}_{i=1}^{n}$, ie, $\mathbb{Z}\left[U_{1}, U_{1}^{-1}, \ldots, U_{n}, U_{n}^{-1}\right]$. Let $H^{!}(G)$ be the homology of $C^{!}(G)$, which is a module over $\mathbb{Z}\left[U, U^{-1}\right]$. 
We will find it useful to use the following:

Definition 6.8 A domain $p$ from $\mathbf{x}$ to $\mathbf{y}$ is a two-chain in $T^{2}$ whose boundary $\partial p$ is a path from $\mathbf{x}$ to $\mathbf{y}$. We denote the set of domains from $\mathbf{x}$ to $\mathbf{y}$ by $\pi(\mathbf{x}, \mathbf{y})$. Given $\phi \in \pi(\mathbf{x}, \mathbf{y})$, let $X_{j}(\phi)$, resp. $O_{j}(\phi)$, denote the local multiplicity of $\phi$ at $X_{j}$, resp. $O_{j}$.

Lemma 6.9 $H^{!}(G)$ is isomorphic to $\mathbb{Z}\left[U, U^{-1}\right]$, and it is generated by $\left[\boldsymbol{x}^{+}(G)\right]$ or $\left[x^{-}(G)\right]$.

Proof The chain complexes $C^{!}$for different positions of the $O$ 's are isomorphic, as follows. Suppose that $G$ is a grid diagram with grid number $m$, with two alternate possible placement of the $O$ 's: $\mathbb{O}$ and $\mathbb{O}^{\prime}$, and let $C^{!}(G)$ and $C^{!}\left(G^{\prime}\right)$ be the two possible corresponding chain complexes. We will construct an isomorphism $\Phi: C^{!}(G) \rightarrow C^{!}\left(G^{\prime}\right)$.

Fix $\mathbf{x}_{0} \in \mathbf{S}(G)$ arbitrarily, let $\mathbf{z}_{0}$ and $\mathbf{z}_{0}^{\prime}$ be the corresponding elements of $C^{!}(G)$ and $C^{!}\left(G^{\prime}\right)$, and declare $\Phi\left(\mathbf{z}_{0}\right)=\mathbf{z}_{0}^{\prime}$. Given any generator of $C^{!}(G)$ as a $\mathbb{Z}\left[U_{1}, U_{1}^{-1}\right]$ module, which has the form of $U_{2}^{n_{2}} \cdots U_{m}^{n} \cdot \mathbf{y}$, it is easy to see that there is a unique $\phi \in \pi(\mathbf{x}, \mathbf{y})$ with $X_{j}(\phi)=0$ for all $j$, and $O_{j}(\phi)=n_{j}$ for $j=2, \ldots, m$. (We use here the fact that $G$ represents a knot, rather than a link with more than one component.)

We then define $\Phi\left(U_{2} \cdots U_{m} \cdot \mathbf{y}\right)=U_{2}^{O_{2}^{\prime}(\phi)} \cdots U_{m}^{O_{m}^{\prime}(\phi)} \cdot \mathbf{y}$. This is easily seen to be an isomorphism of chain complexes of $\mathbb{Z}\left[U, U^{-1}\right]$-modules, where we take $U=U_{1}$.

In particular the complex $C^{!}(G)$ can be identified with the complex $C^{!}\left(G^{\prime}\right)$, where $G^{\prime}$ is the grid diagram with one $O$ directly beneath each $X$; note that $G^{\prime}$ is a diagram for the unknot. Furthermore, note that $\left[z^{+}\left(G^{\prime}\right)\right]$ in $H^{!}\left(G^{\prime}\right)$ is invariant up to units under all destabilizations, by Theorems 1.3 (since multiplication by $U_{i}$ is now invertible). Therefore we can simplify $G^{\prime}$ until we get a $2 \times 2$ grid diagram $G_{0}$ representing a trivial unknot, where an elementary calculation shows $H^{!}\left(G_{0}\right)$ is rank one, generated by $\left[z^{+}\left(G_{0}\right)\right]$. Again, similar computations work for $\boldsymbol{x}^{-}(G)$.

Proof of Theorem 1.5 By Lemma 6.9, the map from $H F K^{-}(G)$ to $H^{!}(G)$ takes $\lambda^{+}(G)$ to a generator of $H^{!}(G)$. In particular $U^{m} \lambda^{+}(G) \neq 0$ for any nonnegative integer $m$.

We can now complete Definition 6.1.

Definition 6.10 The signs in the definition of $z^{-}(G)$ and $z^{+}(G)$ in Definition 6.1 are chosen so that $z^{+}(G)=x^{+}(G)$, and there is an $m \in \mathbb{Z}$ so that $\left[z^{-}(G)\right]=U^{m}\left[z^{+}(G)\right]$ when thought of as elements of $H^{!}(G)$. 
Proof of Theorem 1.1, with signs With the above definition, we can see that in Lemmas 6.5 and 6.6, either $z^{+}(G)$ and $z^{-}(G)$ map to $z^{+}(H)$ and $z^{-}(H)$ respectively, or they map to $-z^{+}(H)$ and $-z^{-}(H)$, depending on whether the chosen generator of $H^{!}(G)$ maps to the chosen generator of $H^{!}(H)$ or its negative. By negating the chain map $\Phi$ from $C^{-}(G)$ to $C^{-}(H)$ if necessary, we can make it map $z^{+}(G)$ to $z^{+}(H)$ and $z^{-}(G)$ to $z^{-}(H)$, with correct signs.

Similarly we can fix the signs in Proposition 1.2 and Theorem 1.3.

\section{The case of links}

Most of the discussion from the earlier parts of this paper have rather straightforward generalizations to the case of links, as we will now show.

Let $\overrightarrow{\mathcal{L}}$ be an oriented Legendrian link with $\ell$ components. The contact distribution $\xi$ determines a complex line bundle over $S^{3}$ equipped with a trivialization in a neighborhood of $\overrightarrow{\mathcal{L}}$. The Euler class of this line bundle relative to its trivialization on the boundary gives an element of $H^{2}\left(S^{3}, \overrightarrow{\mathcal{L}} ; \mathbb{Z}\right) \cong H_{1}\left(S^{3}-L ; \mathbb{Z}\right) \cong \mathbb{Z}^{\ell}$, which plays the role of the rotation number. More concretely, we obtain $\ell$ integers, $\mathrm{r}_{i}, i=1 \ldots \ell$, determined by the property that if $F$ is any surface in $S^{3}$ whose boundary lies on $\overrightarrow{\mathcal{L}}$, then

$$
\left\langle e\left(\left.\xi\right|_{\partial F}, \overrightarrow{\mathcal{L}}^{\prime}\right),[F]\right\rangle=\sum_{i=1}^{\ell} \mathrm{r}_{i} \cdot \#\left(F \cap m_{i}\right),
$$

where $\left\{m_{i}\right\}_{i=1}^{\ell}$ are the meridians for the components of $L$. Similarly, the ThurstonBennequin framing gives an element of $H_{1}\left(S^{3}-L ; \mathbb{Z}\right)$, which we can write as

$$
\sum_{i=1}^{\ell} \mathrm{tb}_{i} \cdot m_{i}
$$

That is, $\mathrm{tb}_{i}$ is the linking number of the Legendrian pushoff of $\overrightarrow{\mathcal{L}}$ with the $i$-th component of $\overrightarrow{\mathcal{L}}$. Note that $\mathrm{tb}_{i}$ depends on the orientation of $\overrightarrow{\mathcal{L}}$. One alternate (equivalent) data to $\mathrm{tb}_{i}$ is the Thurston-Bennequin invariant of the $i$-th component considered as a knot by itself; that choice is less convenient for us.

In terms of the front projection $\Pi=\bigcup_{i=1}^{\ell} \Pi_{i}$, we have that

$$
\begin{aligned}
\operatorname{tb}_{i}(\overrightarrow{\mathcal{L}}) & =\operatorname{wr}\left(\Pi_{i}\right)+\operatorname{lk}\left(\Pi_{i}, \Pi-\Pi_{i}\right)-\frac{1}{2} \#\left\{\text { cusps in } \Pi_{i}\right\} \\
\mathrm{r}_{i}(\overrightarrow{\mathcal{L}}) & =\frac{1}{2}\left(\#\left\{\text { downward-oriented cusps in } \Pi_{i}\right\}\right. \\
& \left.-\#\left\{\text { upward-oriented cusps in } \Pi_{i}\right\}\right) .
\end{aligned}
$$


Let $G$ be a grid diagram for $\overrightarrow{\mathcal{L}}$. We can, of course, define the chain complex $C^{-}(G)$ as before, but in fact, this complex also has a refinement. Specifically, we can consider the Alexander multigrading, which is a function $A: \mathbf{S} \rightarrow \mathbb{Z}^{\ell}$ defined as follows. We partition $\mathbb{O}$ as $\bigcup_{i=1}^{\ell} \mathbb{O}_{i}$ and $\mathbb{X}$ as $\bigcup_{i=1}^{\ell} \mathbb{X}_{i}$, where $\mathbb{O}_{i}$ (respectively $\mathbb{X}_{i}$ ) denotes the set of $O_{j}$ (respectively $X_{j}$ ) corresponding to the $i$-th component of the link. Let $n_{i}=\# \mathbb{O}_{i}$. Now we can define $A(\mathbf{x})=\left(A_{1}(\mathbf{x}), \ldots, A_{\ell}(\mathbf{x})\right)$, where

$$
A_{i}(\mathbf{x})=\mathcal{J}\left(\mathbf{x}-\frac{1}{2}(\mathbb{X}+\mathbb{O}), \mathbb{X}_{i}-\mathbb{O}_{i}\right)-\left(\frac{n_{i}-1}{2}\right) .
$$

We can form the chain complex $C L^{-}(\vec{L})$, defined as in Equation (7). We number the variables so that the first $\ell$ of the $O$ 's, $O_{1}, \ldots, O_{\ell}$, belong to the $\ell$ different components of the link. Taking the homology of this module, we obtain a graded module over $\mathcal{R}=\mathbb{Z}\left[U_{1}, \ldots, U_{\ell}\right]$

$$
H F L^{-}(L)=\bigoplus_{\substack{d \in \mathbb{Z} \\ \mathbf{s} \in \mathbb{Z}^{\ell}}} H F L_{d}^{-}(L, \mathbf{s}),
$$

where $U_{i}$ acts as an endomorphism which is homogeneous of degree -2 for $d$, degree -1 for the $i$-th component of $\mathbf{s}$, and degree 0 otherwise. This $\mathcal{R}$-module is an oriented link invariant [10] which, when specialized to coefficients in $\mathbb{F}_{2}$, agrees with link Floer homology [17].

Definition 6.1 readily generalizes to this context, giving a pair of elements $z^{-}, z^{+} \in$ $C^{-}(G)$, each of which is a cycle in the associated graded object $C L^{-}(G)$. We have the following analogue of Theorem 1.1.

Theorem 7.1 For a grid diagram $G$, let $\overrightarrow{\mathcal{L}}=\overrightarrow{\mathcal{L}}(G)$ be the corresponding oriented Legendrian link. Then there are two associated cycles $z^{+}=z^{+}(G)$ and $z^{-}=z^{-}(G)$, supported in gradings

$$
\begin{array}{ll}
A_{j}\left(z^{+}\right)=\frac{\mathrm{tb}_{j}(\overrightarrow{\mathcal{K}})-\mathrm{r}_{j}(\overrightarrow{\mathcal{K}})+1}{2} & A_{j}\left(z^{-}\right)=\frac{\mathrm{tb}_{j}(\overrightarrow{\mathcal{K}})+\mathrm{r}_{j}(\overrightarrow{\mathcal{K}})+1}{2} \\
M\left(z^{+}\right)=2 \sum_{j=1}^{\ell} A_{j}\left(z^{+}\right)+1-\ell & M\left(z^{-}\right)=2 \sum_{j=1}^{\ell} A_{j}\left(z^{-}\right)+1-\ell,
\end{array}
$$

where here $\ell$ denotes the number of components of $\overrightarrow{\mathcal{L}}$. Moreover, if $G$ and $G^{\prime}$ are two different grid diagrams which represent Legendrian isotopic oriented links, then there is a quasi-isomorphism of chain complexes

$$
\Phi: C^{-}(G) \longrightarrow C^{-}\left(G^{\prime}\right)
$$

with

$$
\Phi\left(z^{+}(G)\right)=z^{+}\left(G^{\prime}\right) \quad \Phi\left(z^{-}(G)\right)=z^{-}\left(G^{\prime}\right) .
$$


Proof Most of this proof is a straightforward generalization of the proof of Theorem 1.1. For example, Equation (19) is a straightforward adaptation of the argument from Lemma 6.3.

Equation (18) follows along the lines of the proof of Lemma 6.4, but with a little extra care. We first argue that

$$
2 A_{j}\left(z^{+}\right)=-\operatorname{wr}(K)-\#\{\text { downward cusps on component } j\}+1 .
$$

As in Lemma 6.4, we consider the horizontal arc $K_{i}$ connecting $X_{i}$ and $O_{i}$, and the corresponding partition of the plane into regions $A, B, C$, and $D$. We also let $K_{i}$ denote the corresponding horizontal strip in the plane. We then analyze the contributions to $2 \mathcal{J}\left(K_{i} \cap\left(x^{+}-\frac{1}{2}(\mathbb{X}+\mathbb{O})\right),\left(\mathbb{X}_{j}-\mathbb{O}_{j}\right)\right)$ coming from the portion of $\left(\mathbb{X}_{j}-\mathbb{O}_{j}\right)$ in these four possible regions.

As in Equation (12), we have

$$
2 \mathcal{J}\left(K_{i} \cap\left(\boldsymbol{x}^{+}-\frac{1}{2}(\mathbb{X}+\mathbb{O})\right), D \cap\left(\mathbb{X}_{j}-\mathbb{O}_{j}\right)\right)=0 .
$$

As in Equation (13), provided that $K_{i} \subset L_{j}$, we have

$$
\begin{aligned}
& 2 \mathcal{J}\left(K _ { i } \cap \left(x^{+}-\right.\right.\left.\left.\frac{1}{2}(\mathbb{X}+\mathbb{O})\right), C \cap\left(\mathbb{X}_{j}-\mathbb{O}_{j}\right)\right) \\
&=\#\left\{\text { negative crossings of } L_{j} \text { with } K_{i}\right\} \\
& \quad-\#\left\{\text { positive crossings of } L_{j} \text { with } K_{i}\right\} .
\end{aligned}
$$

Finally, we consider the analogue of Equation (14). If $K_{i}$ is an arc on component $j$, by considering the eight cases from Figure 8 , we have that

$$
\begin{aligned}
2 \mathcal{J}\left(K_{i} \cap\left(x^{+}-\frac{1}{2}(\mathbb{X}+\mathbb{O})\right),(A\right. & \left.\cup B) \cap\left(\mathbb{X}_{j}-\mathbb{O}_{j}\right)\right) \\
=1 & -\#\left\{\text { downward cusps among }\left\{X_{i}, O_{i}\right\}\right\} \\
& -\left(\frac{1}{2} \text { if } \mathbb{O} \cap K_{i} \text { is above the } X \text { in its column }\right) \\
& +\left(\frac{1}{2} \text { if } \mathbb{X} \cap K_{i} \text { is below the } O \text { in its column }\right) .
\end{aligned}
$$

Otherwise, this contribution is zero. We deduce Equation (20) by adding up half the local contributions calculated above and subtracting $\left(n_{i}-1\right) / 2$, as in Equation (17). The statement for $A_{j}\left(x^{+}\right)$follows now from that equation, together with Equations (15) and (16).

The definition of $\boldsymbol{x}^{+}$and $\boldsymbol{x}^{-}$is as before. The proof of invariance under the chain map up to signs follows identically. The signs in the definition of $z^{+}$and $z^{-}$are fixed later, in Definition 7.5. 
Let $\lambda^{+}(\overrightarrow{\mathcal{L}})$ and $\lambda^{-}(\overrightarrow{\mathcal{L}})$ denote the homology classes in $\operatorname{HFL}^{-}(L)$ of $z^{+}(G)$ and $z^{-}(G)$. These are Legendrian invariants of $\overrightarrow{\mathcal{L}}$. Behavior under orientation reversal of all components simultaneously and Legendrian mirror is the same as in Proposition 1.2. The analogue of Theorem 1.3 is the following.

Theorem 7.2 Let $\overrightarrow{\mathcal{L}}$ be an oriented Legendrian link, and $\overrightarrow{\mathcal{L}}^{-}$(respectively $\overrightarrow{\mathcal{L}}^{+}$) be oriented Legendrian links obtained as a single negative (respectively positive) stabilization of $\overrightarrow{\mathcal{L}}$ on the $j$-th component. Then there is a quasi-isomorphism

$$
\begin{aligned}
& \Phi^{-}: C(\overrightarrow{\mathcal{L}}) \longrightarrow C\left(\overrightarrow{\mathcal{L}}^{-}\right) \\
& \Phi^{+}: C(\overrightarrow{\mathcal{L}}) \longrightarrow C\left(\overrightarrow{\mathcal{L}}^{+}\right)
\end{aligned}
$$

under which

$$
\begin{aligned}
\Phi^{-}\left(\lambda^{+}(\overrightarrow{\mathcal{L}})\right) & =\lambda^{+}\left(\overrightarrow{\mathcal{L}}^{-}\right) & U_{j} \cdot \Phi^{-}\left(\lambda^{-}(\overrightarrow{\mathcal{L}})\right) & =\lambda^{-}\left(\overrightarrow{\mathcal{L}}^{-}\right) \\
U_{j} \cdot \Phi^{+}\left(\lambda^{+}(\overrightarrow{\mathcal{L}})\right) & =\lambda^{+}\left(\overrightarrow{\mathcal{L}}^{+}\right) & \Phi^{+}\left(\lambda^{-}(\overrightarrow{\mathcal{L}})\right) & =\lambda^{-}\left(\overrightarrow{\mathcal{L}}^{+}\right) .
\end{aligned}
$$

Proof This is a straightforward generalization of the proof of Theorem 1.3.

Similarly, we have the following generalization of Theorem 1.5:

Theorem 7.3 For a Legendrian link $\overrightarrow{\mathcal{L}}$, the homology classes $\lambda^{+}(\overrightarrow{\mathcal{L}})$ and $\lambda^{-}(\overrightarrow{\mathcal{L}})$ are nontrivial; and indeed, they are not $U_{i}$-torsion for any of the $U_{i}$.

Proof A little more care is needed than in the case of knots: $H^{!}(G)$ now does depend on the placement of the $O_{i}$. However, if we consider the new complex $C^{\prime}(G)=$ $C^{-}(G) /\left\{U_{i}=1\right\}_{i=1}^{m}$, then the homology of this complex $H^{\prime}(G)$ obviously no longer depends on the placement of the $O_{i}$ (since they play no role in the $C^{\prime}(G)$ ). Thus we can move placements of the $\mathbb{O}$ to realize the unknot. But we already know by Theorem 1.5 that the invariants for the unknot do not vanish in $\mathrm{HFK}^{-}$, so they do not vanish in $H^{\prime}(G)$, so both $\lambda^{+}(\overrightarrow{\mathcal{L}})$ and $\lambda^{-}(\overrightarrow{\mathcal{L}})$ represent nontrivial homology classes in $H^{\prime}(G)$. It follows readily that the two classes are not $U_{i}$-torsion for any of the $U_{i}$.

As a final point in this section, we turn to the signs entering the definition of $\lambda^{+}(L)$ and $\lambda^{-}(L)$, generalizing Definition 6.10 to the case of links.

Let $C^{\prime \prime}(G)=C(G) /\left\{U_{1}=\cdots=U_{\ell}=1\right\}$, where as usual we number the $U_{i}$ so that the first $\ell$ correspond to the $\ell$ distinct components of the link. Although the complex no longer inherits neither an Alexander of Maslov gradings, $C^{\prime \prime}$ retains an integral grading given by $N=M-2 A+\ell-1$. 
Lemma 7.4 We have that $H_{*}\left(C^{\prime \prime}(G)\right) \cong H_{*}\left(T^{\ell-1}\right)$, and the elements $\left[x^{+}(G)\right]$ and $\left[x^{-}(G)\right]$ generate the zero-dimensional part.

Proof Let $C^{\prime}(G)$ denote the complex studied in the proof of Theorem 7.3. As in that proof, its homology is independent of the placement of the $O_{i}$, so it is isomorphic to the value for the unknot, from which it is easy to see that $H_{*}\left(C^{\prime}(G)\right) \cong H_{*}\left(T^{m-1}\right)$ with an appropriate overall grading shift. (Remember that $H_{*}\left(T^{m-1}\right)$ is a $(m-1)$-fold tensor product of a space $V^{\prime}$ with itself, where $V^{\prime} \cong \mathbb{Z} \oplus \mathbb{Z}$ with one generator in degree 0 and one in degree 1.)

Note that $C^{\prime}(G)=C^{\prime \prime}(G) /\left\{U_{\ell+1}=\cdots=U_{m}=1\right\}$. Moreover, in $C^{\prime}(G)$, each $U_{i}$ is homotopy equivalent to multiplication by 1 . (This follows from the fact that in $C L^{-}$, multiplication by $U_{i}$ and multiplication by $U_{j}$ are chain homotopic if $O_{i}$ and $O_{j}$ correspond to the same component of the link; see [10, Lemma 2.9], together with the fact that we have set $U_{i}=1$ for $i=1, \ldots, \ell$ in $C^{\prime \prime}(G)$.) Thus, it follows that $H_{*}\left(C^{\prime}(G)\right) \cong H_{*}\left(C^{\prime \prime}(G)\right) \otimes H_{*}\left(T^{m-\ell}\right)$. Combining this with the fact that $H_{*}\left(C^{\prime}(G)\right) \cong H_{*}\left(T^{m-1}\right)$, we can conclude that $H_{*}\left(C^{\prime \prime}(G)\right) \cong H_{*}\left(T^{\ell-1}\right)$.

From the proof of Theorem 7.3, $\left[x^{+}(G)\right]$ and $\left[x^{-}(G)\right]$ are primitive elements in $H_{*}\left(C^{\prime \prime}(G)\right)$. From Equation (19), we conclude that they both are supported in degree zero.

In view of Lemma 7.4, can generalize Definition 6.10 to the case of links, as follows.

Definition 7.5 The signs in the definition of $z^{+}$and $z^{-}$are chosen so that $\left[z^{+}(G)\right]$ and $\left[z^{-}(G)\right]$ represent the same class in the homology of $C^{\prime \prime}(G)=C(G) /\left\{U_{1}=\cdots=\right.$ $\left.U_{\ell}=1\right\}$.

This is clearly compatible with Definition 6.10 in the case where $\ell=1$, and as before we can now fix the signs in Theorems 7.1 and 7.2.

\section{Examples: The knot $5_{2}$ and the link $6_{3}^{2}$}

To demonstrate that our invariant is nontrivial, we will use it to distinguish different Legendrian representatives of the knot $5_{2}$ and the link $6_{3}^{2}$. Throughout this section we will work over $\mathbb{F}_{2}$. 
Example 8.1 The unoriented Legendrian knots $\mathcal{K}_{1}$ and $\mathcal{K}_{2}$ with front projections

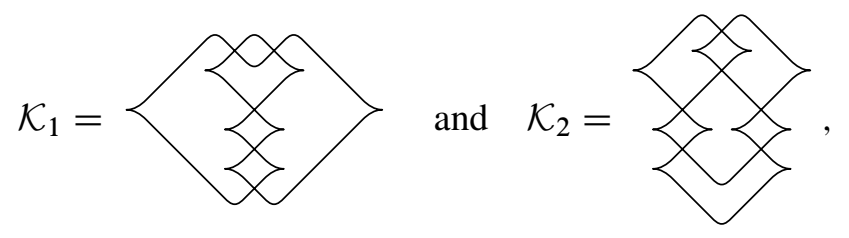

both of topological type $5_{2}$ and having $t b=1$ and $r=0$, are not Legendrian isotopic.

This example was first found by Chekanov [1].

Proof Reflecting through the vertical axis takes both front projections shown above to themselves with orientation reversed. As in Lemma 4.6, reflecting the front through the vertical axis corresponds to rotating the Legendrian knot by $180^{\circ}$ around the $z$-axis, which is an isotopy. Therefore the two oriented Legendrian knots are both isotopic to their reverse and it suffices to prove the result for one orientation.

Grid diagrams $G_{1}, G_{2}$ for the two Legendrian knots are shown in Figure 12. On $G_{1}$, we have indicated the generator $z^{+}$. From the diagram it is straightforward to check that $z^{+}$is isolated in the chain complex: for all generators $\mathbf{x}$, there are no rectangles in $\operatorname{Rect}^{\circ}\left(\mathbf{x}, z^{+}\right)$with empty intersection with $\mathbb{X}$ and $\mathbb{O}$. By the symmetry of the diagram, the same is true for $z^{-}$. It follows that $\lambda^{+}\left(\overrightarrow{\mathcal{K}}_{1}\right) \neq \lambda^{-}\left(\overrightarrow{\mathcal{K}}_{1}\right)$.
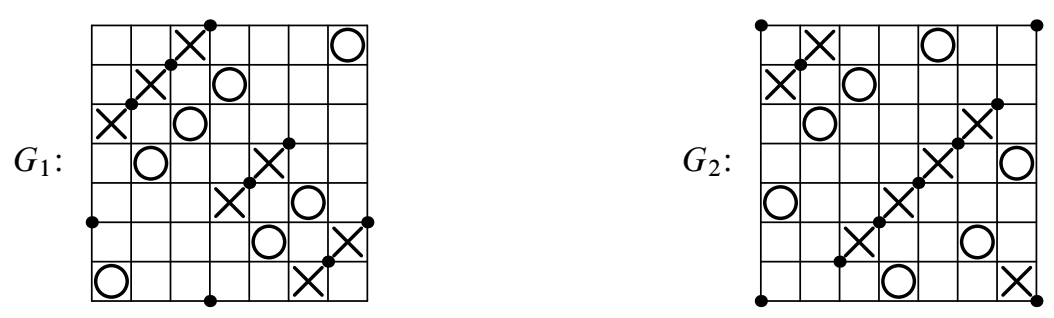

Figure 12: Grid diagrams for two different Legendrian representatives of the knot $5_{2}$. The left diagram shows the generator $z^{+}$. The right diagram shows a generator $\mathbf{y}$ so that $\partial \mathbf{y}=z^{+}+z^{-}$.

On the other hand, consider the generator $\mathbf{y}$ shown on $G_{2}$ in Figure 12. An elementary check shows that $\partial(\mathbf{y})=z^{+}+z^{-}$; therefore $\lambda^{+}\left(\overrightarrow{\mathcal{K}}_{2}\right)=\lambda^{-}\left(\overrightarrow{\mathcal{K}}_{2}\right)$. But if $\overrightarrow{\mathcal{K}}_{1}$ and $\overrightarrow{\mathcal{K}}_{2}$ were Legendrian isotopic (with either orientation), by Theorem 1.1 there would be an isomorphism from $H F K^{-}\left(\overrightarrow{\mathcal{K}}_{1}\right)$ to $H F K^{-}\left(\overrightarrow{\mathcal{K}}_{2}\right)$ taking $\lambda^{+}\left(\overrightarrow{\mathcal{K}}_{1}\right)$ to $\lambda^{+}\left(\overrightarrow{\mathcal{K}}_{2}\right)$ and $\lambda^{-}\left(\overrightarrow{\mathcal{K}}_{1}\right)$ to $\lambda^{-}\left(\overrightarrow{\mathcal{K}}_{2}\right)$, a contradiction. 
Remark 8.2 This example shows that the transverse invariant $\theta(\mathcal{T})$ is only an invariant up to quasi-isomorphisms (and not as an element of an abstract homology group). In particular, a sequence of elementary moves from a grid diagram $G$ back to itself induces a quasi-isomorphism that need not be the identity. Indeed, an elementary calculation shows that the knots $\overrightarrow{\mathcal{K}}_{1}$ and $\overrightarrow{\mathcal{K}}_{2}$ become Legendrian isotopic after either one positive or one negative stabilization. If we follow a path of grid diagrams that takes $G_{1}$ to $G_{2}$ via a positive stabilization and destabilization, followed by a path that takes $G_{2}$ to $G_{1}$ via a negative stabilization and destabilization, we first take $\lambda^{-}\left(\overrightarrow{\mathcal{K}}_{1}\right)$ to $\lambda^{-}\left(\overrightarrow{\mathcal{K}}_{2}\right)$, which is equal to $\lambda^{+}\left(\overrightarrow{\mathcal{K}}_{2}\right)$, which we take to $\lambda^{+}\left(\overrightarrow{\mathcal{K}}_{2}\right)$. Since $\lambda^{-}\left(\overrightarrow{\mathcal{K}}_{1}\right) \neq \lambda^{+}\left(\overrightarrow{\mathcal{K}}_{2}\right)$, this sequence of grid moves induces a nontrivial automorphism of $\operatorname{HFK}^{-}\left(\overrightarrow{\mathcal{K}}_{1}\right)$.

Example 8.3 The oriented Legendrian links $\overrightarrow{\mathcal{L}}_{3}$ and $\overrightarrow{\mathcal{L}}_{4}$ with front projections

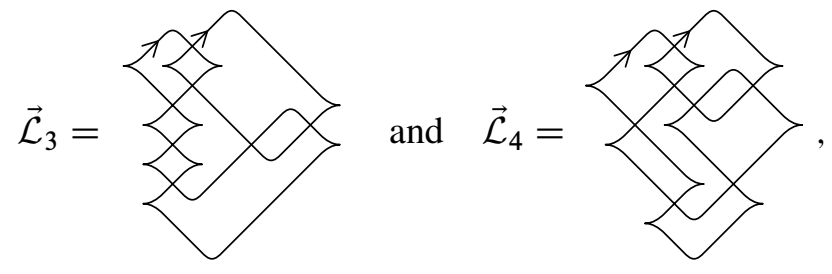

both of topological type $6_{3}^{2}$ and having $\mathrm{tb}_{i}=1$ and $\mathrm{r}_{i}=0$ for $i=1,2$, are not Legendrian isotopic.

Proof Grid diagrams $G_{3}$ and $G_{4}$ for these two links are shown in Figure 13. It is easy to verify that $z^{+}\left(G_{3}\right)$ and $z^{-}\left(G_{3}\right)$ are isolated in the chain complex, as for $G_{1}$. It follows that $\lambda^{+}\left(\overrightarrow{\mathcal{L}}_{3}\right) \neq \lambda^{-}\left(\overrightarrow{\mathcal{L}}_{3}\right)$. On the other hand, we have indicated a generator $\mathbf{y}$ on $G_{4}$ so that $\partial(\mathbf{y})=z^{+}+z^{-}$, so $\lambda^{+}\left(\overrightarrow{\mathcal{L}}_{4}\right)=\lambda^{-}\left(\overrightarrow{\mathcal{L}}_{4}\right)$.
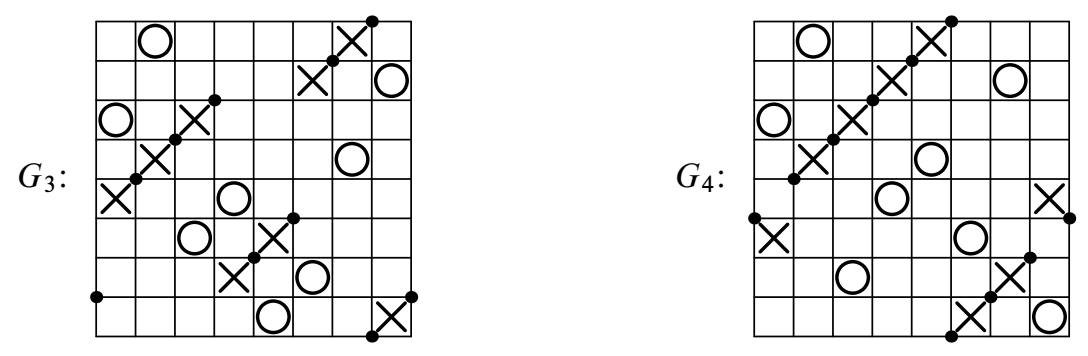

Figure 13: Grid diagrams $G_{3}$ and $G_{4}$ for $\overrightarrow{\mathcal{L}}_{1}$ and $\overrightarrow{\mathcal{L}}_{2}$. The left diagram shows $z^{+}\left(G_{3}\right)$. The right diagram shows a generator $\mathbf{y}$ with $\partial \mathbf{y}=z^{+}+z^{-}$. 


\section{Appendix A On $\tau$}

We found it convenient to work with $H F K^{-}$in the present paper. In particular, in the introduction, we gave a definition of $\tau$ which refers to $H F K^{-}$, ie, it is defined in terms of the associated graded object for the Alexander filtration. By contrast, the usual definition of $\tau$ refers to the filtered complex $\widehat{C F}$ [14]. For completeness, we repeat this definition, putting it in terms of grid diagrams.

Consider the chain complex $C^{-}(G)$ as in Equation (6). This chain complex has a $\mathbb{Z}$ filtration, induced by the Alexander filtration, whose associated graded object is $C K^{-}(G)$ considered throughout most of this paper.

We will need two constructions, as follows. If we set $U_{1}=0$, we obtain a new chain complex $\widehat{C}(G)$, filtered by subcomplexes $\widehat{\mathcal{F}}(K, s)$, which are generated by elements with Alexander grading $\leq s$. Following [14], we define

$$
\tau(K)=\min \left\{s \mid \widehat{\mathcal{F}}(K, s) \longrightarrow \widehat{H F}\left(S^{3}\right) \text { is nontrivial }\right\} .
$$

More symmetrically, we can consider the filtration $\widetilde{\mathcal{F}}(K, s)$ on

$$
\widetilde{C}(G)=C^{-}(G) /\left(U_{1}=\cdots=U_{n}=0\right),
$$

where $\widetilde{\mathcal{F}}(K, s)$, once again, is generated by those intersection points $\mathbf{x}$ with $A(\mathbf{x}) \leq s$. This, too, could be used to calculate $\tau$ : Define

$$
\tilde{\tau}(K)=\min \left\{s \mid H_{*}(\widetilde{\mathcal{F}}(K, s)) \longrightarrow H_{*}(\widetilde{C}(G)) \text { is nontrivial }\right\} .
$$

Lemma A.1 $\tilde{\tau}(K)=\tau(K)-n+1$.

Proof The chain complex $\widetilde{C}(G)$ is filtered quasi-isomorphic to the filtered mapping cone of an iterated mapping cylinder

$$
\left(\frac{C(G)}{U_{1}=0}\right) \otimes_{\mathcal{R}}\left(\bigotimes_{i \neq 1} \mathcal{R}[-1,-1] \stackrel{U_{i}}{\longrightarrow} \mathcal{R}\right),
$$

where $\mathcal{R}=\mathbb{Z}\left[U_{1}, \ldots, U_{n}\right]$, and $\mathcal{R}[-1,-1]$ denotes $\mathcal{R}$ with a shift in bigrading so that 1 has both Alexander filtration and Maslov grading of -1 (see [10, Lemma 2.1]). Since multiplication by $U_{i}$ on $C$ is filtered chain homotopic to multiplication by $U_{1}$ (which we have set equal to zero in the quotient complex), the above mapping cylinder is in fact filtered quasi-isomorphic to

$$
\left(\frac{C}{U_{1}=0}\right) \otimes V^{n-1}
$$


where $V$ is a free Abelian group generated by two elements, one with Maslov and Alexander bigrading $(0,0)$, and another with Maslov and Alexander bigrading $(-1,-1)$. In particular, if we always choose the second generator of $V$, we get a subcomplex of $\widetilde{C}(G)$ which is isomorphic to $\widehat{C}(G)$ with both gradings shifted by $-n+1$. The element with minimal Alexander filtration mapping nontrivially to $H_{*}(\widetilde{C}(G))$ will always live in this subcomplex.

To fit the definition of $\tau$ above into the discussion from the introduction, define

$$
\tau^{\prime}(K)=\max \left\{s \in \mathbb{Z} \mid \exists \xi \in H F K^{-}(K, s) \text { such that } \forall d \geq 0, U^{d} \xi \neq 0\right\} .
$$

Lemma A.2 $\tau^{\prime}(K)=\tau(m(K))$.

Proof Let $C^{\prime}(G)=C^{-}(G) \otimes \mathbb{Z}[U]$, where all $U_{i}$ act on $\mathbb{Z}[U]$ by multiplication by $U$. We think of $C^{\prime}(G)$ as a bigraded complex (rather than as a filtered one), writing $C^{\prime}(G)=\bigoplus_{s \in \mathbb{Z}} C^{\prime}(G, s)$, where here $s$ refers to the Alexander grading. The homology of $C^{\prime}(G)$ is easily seen to agree with $H F^{-}(G) \otimes V^{n-1}$ (following Lemma A.1), where the tensor product is taken in the bigraded sense and, as before, $V$ is a rank two module generated by two elements, one with Maslov and Alexander bigrading $(0,0)$, and another with Maslov and Alexander bigrading $(-1,-1)$. It follows readily that

$$
\tau^{\prime}(K)=\max \left\{s \in \mathbb{Z} \mid \exists \xi \in H_{*}\left(C^{\prime}(G, s)\right) \text { such that } \forall d \geq 0, U^{d} \xi \neq 0\right\} .
$$

Given any $s \in \mathbb{Z}, C^{\prime}(G, s)$ is a chain complex over $\mathbb{Z}$ which still retains its Maslov grading. It is generated by elements $U^{m} \cdot \mathbf{x}$ with $m \geq 0$ and $A(\mathbf{x})-m=s$, its differentials count those empty rectangles with $\sum_{i} X_{i}(r)=0$, and each rectangle is counted with multiplicity $U^{\sum_{i}} O_{i}(r)$. Let $C^{\prime \prime}(G, s)$ be the chain complex generated over $\mathbb{Z}$ by those $\mathbf{x} \in \mathbf{S}$ with $A(\mathbf{x}) \geq s$ and differential

$$
\partial^{\prime \prime}(\mathbf{x})=\sum_{\mathbf{y} \in \mathbf{S}(G)} \sum_{\substack{r \in \operatorname{Rect}^{\circ}(\mathbf{x}, \mathbf{y}) \\ r \cap \mathbb{X}=\varnothing}} \mathcal{S}(r) \cdot \mathbf{y} .
$$

There is a canonical inclusion of complexes $\iota$ : $C^{\prime \prime}(G, s) \subset C^{\prime \prime}(G, s-1)$. Let $C^{\prime \prime}(G)$ be the union of all $C^{\prime \prime}(G, s)$. For all $s \in \mathbb{Z}$, there is an isomorphism of chain complexes of $\mathbb{Z}$-modules

$\begin{aligned} \phi_{s}: C^{\prime}(G, s) & \longrightarrow C^{\prime \prime}(G, s) \\ \phi_{s}\left(U^{m} \mathbf{x}\right) & =\mathbf{x},\end{aligned}$


which fits into the diagram

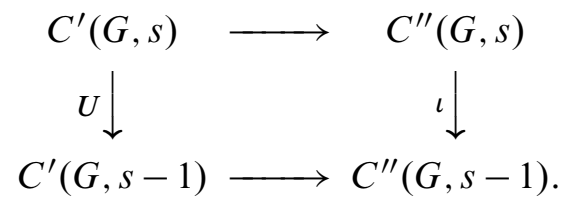

Therefore the inclusion of $H_{*}\left(C^{\prime}(G, s)\right)$ into the direct limit of $H_{*}\left(C^{\prime}(G, *)\right)$ (with connecting map $U)$ corresponds to the inclusion of $H_{*}\left(C^{\prime \prime}(G, s)\right)$ into the homology of $C^{\prime \prime}(G)$.

Let $G^{\prime}$ be the grid diagram obtained by reversing the roles of the $O_{i}$ and the $X_{i}$ in $G$; $G^{\prime}$ is a diagram for $-K$, the knot $K$ with the orientation reversed. Let $A\left(\mathbf{x} ; G^{\prime}\right)$ be the Alexander grading with respect to $G^{\prime}$. By Equation (5) we see that $A\left(\mathbf{x} ; G^{\prime}\right)=$ $-A(\mathbf{x} ; G)-n+1$, so $C^{\prime \prime}(G, s) \cong \mathcal{F}\left(G^{\prime},-s-n+1\right)$. Therefore the filtered chain homotopy type of $C^{\prime \prime}(G)$ is identified with the filtered chain homotopy type of $\widetilde{C}\left(G^{\prime}\right)$, with modified filtration degree. Thus $\tau^{\prime}(K)=-\tilde{\tau}(-K)-n+1$ and, by Lemma A.1, $\tau^{\prime}(K)=-\tau(-K)$. We also have $\tau(K)=\tau(-K)=-\tau(m(K))$ [14]. (This last step can also be proved using grid diagrams alone, $\mathrm{cf}$ [10, Proposition 5.5].) Putting these together, we complete the proof.

\section{References}

[1] Y Chekanov, Differential algebra of Legendrian links, Invent. Math. 150 (2002) 441483 MR1946550

[2] P R Cromwell, Embedding knots and links in an open book. I. Basic properties, Topology Appl. 64 (1995) 37-58 MR1339757

[3] I A Dynnikov, Arc-presentations of links: monotonic simplification, Fund. Math. 190 (2006) 29-76 MR2232855

[4] J Epstein, D Fuchs, M Meyer, Chekanov-Eliashberg invariants and transverse approximations of Legendrian knots, Pacific J. Math. 201 (2001) 89-106 MR1867893

[5] J B Etnyre, Legendrian and transversal knots, from: "Handbook of knot theory", Elsevier B. V., Amsterdam (2005) 105-185 MR2179261

[6] J B Etnyre, K Honda, Knots and contact geometry. I. Torus knots and the figure eight knot, J. Symplectic Geom. 1 (2001) 63-120 MR1959579

[7] D Fuchs, S Tabachnikov, Invariants of Legendrian and transverse knots in the standard contact space, Topology 36 (1997) 1025-1053 MR1445553

[8] P B Kronheimer, T S Mrowka, Gauge theory for embedded surfaces. I, Topology 32 (1993) 773-826 MR1241873 
[9] C Manolescu, P Ozsváth, S Sarkar, A combinatorial description of knot Floer homology, to appear in Ann. of Math. (2) arXiv:math.GT/0607691

[10] C Manolescu, P Ozsváth, Z Szabó, D Thurston, On combinatorial link Floer homology, Geom. Topol. 11 (2007) 2339-2412 MR2372850

[11] L Ng, Computable Legendrian invariants, Topology 42 (2003) 55-82 MR1928645

[12] L Ng, A Legendrian Thurston-Bennequin bound from Khovanov homology, Algebr. Geom. Topol. 5 (2005) 1637-1653 MR2186113

[13] L Ng, P Ozsváth, Z Szabó, Transverse knots distinguished by knot Floer Homology, to appear in J. Symplectic Geom. arXiv:math.GT/0703446

[14] P Ozsváth, Z Szabó, Knot Floer homology and the four-ball genus, Geom. Topol. 7 (2003) 615-639 MR2026543

[15] P Ozsváth, Z Szabó, Holomorphic disks and knot invariants, Adv. Math. 186 (2004) 58-116 MR2065507

[16] P Ozsváth, Z Szabó, Heegaard Floer homology and contact structures, Duke Math. J. 129 (2005) 39-61 MR2153455

[17] P Ozsváth, Z Szabó, Holomorphic disks, link invariants, and the multi-variable Alexander polynomial, to appear in Algebr. Geom. Topol. 8 (2008) arXiv: math.GT/0512286

[18] O Plamenevskaya, Bounds for the Thurston-Bennequin number from Floer homology, Algebr. Geom. Topol. 4 (2004) 399-406 MR2077671

[19] O Plamenevskaya, Transverse knots and Khovanov homology, Math. Res. Lett. 13 (2006) 571-586 MR2250492

[20] J A Rasmussen, Khovanov homology and the slice genus, to appear in Invent. Math. arXiv:math.GT/0402131

[21] J A Rasmussen, Floer homology and knot complements, $\mathrm{PhD}$ thesis, Harvard University (2003) arXiv:math.GT/0306378

[22] L Rudolph, An obstruction to sliceness via contact geometry and "classical” gauge theory, Invent. Math. 119 (1995) 155-163 MR1309974

Department of Mathematics, Columbia University

New York, NY 10027

Department of Mathematics, Princeton University

Princeton, New Jersey 08544

Department of Mathematics, Barnard College, Columbia University New York, NY 10027

petero@math.columbia.edu, szabo@math.princeton.edu, dthurston@barnard.edu 
Proposed: Yasha Eliashberg

Received: 21 February 2007

Seconded: Tom Mrowka, John Morgan

Revised: 5 January 2008

Geometry 8 Topology, Volume 12 (2008) 